\title{
Performance evaluation of the tandem C4 blades for axial-flow compressors
}

\author{
Atef Mohamed Alm-Eldien ${ }^{1}$, Ahmed Farouk Abdel Gawad ${ }^{2}$, Gamal Hafaz, \\ Mohamed Gaber Abd El Kreim, ${ }^{3, *}$ \\ ${ }^{1}$ Mech. Power Eng. Dept., Faculty of Eng., Port Said University, Port Said, Egypt \\ ${ }^{2}$ Professor of Computational Fluid Mechanics, Mech. Eng. Dept., Umm Al-Qura Univ., Makkah, Saudi Arabia \\ ${ }^{3}$ Gas Turbine Maintenance Engineer, East Delta Electricity Production Company, Ismailia, Egypt
}

\section{Email address:}

atef_alameldin@yahoo.com (A. M. Alm-Eldien), afaroukg@yahoo.com (A. F. A. Gawad), mgaber1_eg@hotmail.com (M. G. A. E. Kreim)

\section{To cite this article:}

Atef Mohamed Alm-Eldien, Ahmed Farouk Abdel Gawad, Gamal Hafaz, Mohamed Gaber Abd El Kreim. Performance Evaluation of the Tandem C4 Blades for Axial-Flow Compressors. American Journal of Aerospace Engineering. Special Issue: Hands-on Learning Technique for Multidisciplinary Engineering Education. Vol. 2, No. 1-1, 2015, pp. 74-92. doi: 10.11648/j.ajae.s.2015020101.17

\begin{abstract}
The purpose of this work is to study the aerodynamic performance of a tandem C4 base-profile compressor blade using numerical tools. In this paper, the flow along the tandem blade is studied for various relative blade positions. In all the studied cases, the front blade is fixed and the position of the rear blade is varied as a function of the axial and tangential displacements. A computer code was developed in "Visual Basic" using linear strength vortex-panel method to predict the aerodynamic performance of the tandem blade.
\end{abstract}

Keywords: Tandem Blades, Linear Strength Vortex Panel Method, Rotor, Stator, Axial-Compressor

\section{Introduction}

Looking at the Euler Turbomachinery Equation (1), it obviously indicates that there are two ways of achieving high loading at compressor blades. First one is the increase of the rotational speed. Second one is the increase of the tangential-velocity difference between inlet and exit of a compressor rotor. That is increasing the turning angle which is the tandem blade concept.

$$
\frac{-P_{\text {shaft }}}{\dot{m}}=U_{2} V_{\theta 2}-U_{1} V_{\theta 1}
$$

Where, $U_{2}$ is blade speed at radius $r_{2}, U_{1}$ is blade speed at radius $r_{l}, V_{\theta 1}$ is tangential velocity at radius $r_{1}, V_{\theta 2}$ is tangential velocity at radius $r_{2}, \dot{m}$ is mass flow rate $(\mathrm{kg} / \mathrm{s})$, $P_{\text {shaft }}$ is shaft power $(k W)$.

A major limitation on the pressure rise in a subsonic axial flow compressor stage is boundary layer separation on the blade suction surface. One method of mitigating the suction surface separation is to employ tandem blades. Tandem blading is a method of increasing the flow deflection by delaying the separation in diffusing cascade arrangements. The two parts of a blade, the front and the rear, are arranged so that the air from the pressure surface of the forward blade is injected on the suction surface of rear blade. Due to this injection of air the boundary layer on the suction surface gets more momentum to follow the rest of the suction surface. Thus, the separation of the boundary layer is delayed. According to the axial and tangential displacements, the interference area between the blades plays the role of a convergent or a convergent-divergent nozzle-type of passage, through which the air from the pressure surface of the foreword blade blows on the suction surface of the rear blade. The configuration of the front and rear blade is arranged in such a way that the front blade is truncated approximately at the middle and then the rear blade is configured so that the suction surface keeps its continuity of shape to provide the required camber. By induction of extra momentum, the tandem blade increases turning or loading capability of a given blade row. It also gives a wider stall-free operating range and hence efficiency of the compressor can be increased at off-design conditions. All these benefits of tandem blade gives the solution to one of the oldest challenges faced by axial-flow compressor designers; that is to use as few stages as possible to achieve the desired pressure rise without compromising efficiency. The obvious benefits of using fewer stages are the improvement of engine power-to-weight ratio and the reduction in manufacturing parts. 


\section{Literature Review}

Many attempts have been made to understand the flow in a tandem cascade. Experimental as well as analytical approaches are reported for many flow conditions and different blade parameters.

Wennerstrom [1] studied the potential of tandem-airfoil blading for improving the efficiency and stable operating range of compressor stages. The investigation included testing of one single-airfoil blading, one tandem-blade with a $20-80 \%$ loading split and one tandem-blade with 50-50\% loading split (the loading was estimated using the diffusion factor criterion).The results of his effort showed that tandem configuration, with the majority of loading on the rear airfoil should have a larger operation range than conventional blades, was not substantiated. But with 50-50\% loading split between the airfoils, the tandem arrangement demonstrated higher pressure rise and efficiency than the corresponding single one.

Saha and Roy [2],[3] conducted various aerodynamic performance evaluations of a single and a tandem cascade for a wide range of inlet angles. The purpose of the investigation was to determine the high deflection capabilities of the tandem blade and to compare the results at off-design with an equivalent single one. The results of the study showed that the diffusion capabilities of the tandem blade are higher compared to a single airfoil.

Other investigations in tandem airfoils [4],[5] indicated that the flow deflection capabilities shown by the tandem configuration at design point, is higher compared with a single one. This fact has been explained by the formation of a new boundary layer at the rear blade.

\section{Applications of Tandem Blade Rows}

In the practice, the tandem cascades are applied to compressors in the subsonic, transonic and even supersonic range for rotors and stators [5]. But the main use of this arrangement is in the stator of the final stage in axial compressor, where the flow enters with high swirl velocity and it has to be turned to the radial direction. Therefore, the flow-turning angle is rather high and the last stator row is heavily loaded with the danger of flow separation. Figure 1 shows a General Electric heavy-duty gas turbine that uses a triple tandem blade row in the stator of the last stage in the axial compressor [6]. Characteristics of the compressor are shown in table 1.

Table 1. Heavy Duty tandem compressor characteristics

\begin{tabular}{ll}
\hline Manufacturer & General Electric \\
\hline Type & MS 7001 EA \\
Power & $86.2 \mathrm{MW}$ \\
Thermal efficiency $\left(\eta_{t h}\right)$ & $33.0 \%$ \\
Pressure ratio $(\pi)$ & 11.9 \\
Mass flow & $299[\mathrm{~kg} / \mathrm{s}]$ \\
Turbine outlet temperature & 537 \\
Revolutions & $3600 \mathrm{rev} / \mathrm{min}$ \\
\hline
\end{tabular}

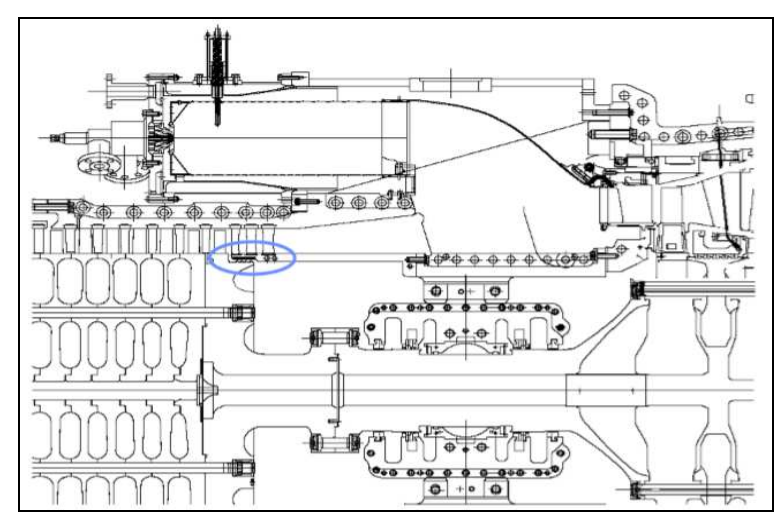

Figure 1. Heavy-duty gas turbine with tandem compressor blades in the last stator row [6].

Till now, tandem rotor concept has applications only for experimental purposes. Figure 2 shows a photo of tandem rotor built at The Institute of Turbomachinery of The Hannover University [7].

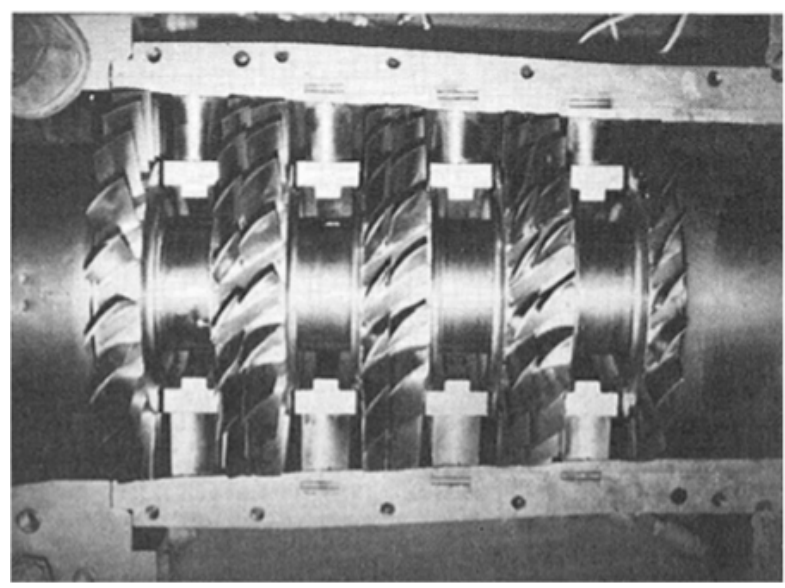

Figure 2. Tandem rotor [7].

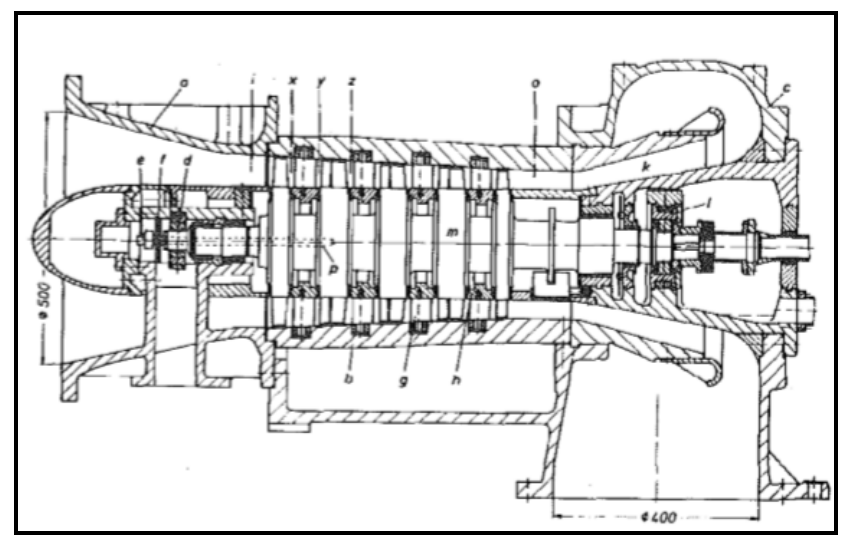

Figure 3. Tandem rotor compressor [7].

One of the first tandem blade studies was performed in Germany [7],[8], Figure 3. This was a 4-stage axial compressor where 3 stages were built as tandem rotors and having a design pressure ratio of 2.5. Detailed characteristics of the experimental axial compressor are listed in Table 2.

Other interesting studies of tandem blades can be found in [9]-[11]. 
Table 2. Experimental set-up characteristic

\begin{tabular}{lll}
\hline Name & Value & Units \\
\hline Mass flow rate & 6.58 & $\mathrm{~kg} / \mathrm{s}$ \\
Speed & 11000 & $\mathrm{rpm}$ \\
Number of stages & 4 & - \\
Design pressure ratio & 2.5 & - \\
Hub to tip ratio & 0.64 & - \\
Blade tip speed & 195.82 & $\mathrm{~m} / \mathrm{s}$ \\
Internal power & 672 & $\mathrm{~kW}$ \\
Inlet total pressure & 1 & $\mathrm{Bar}$ \\
Inlet total temperature & 288.15 & $\mathrm{~K}$ \\
Outlet total pressure & 2.51 & $\mathrm{Bar}$ \\
Outlet total temperature & 389.9 & $\mathrm{~K}$ \\
\hline
\end{tabular}

\section{Geometry Description}

The geometry and aerodynamic parameters for a tandem blade row are almost the same as those used for single airfoils, but two additional variables appear in the arrangement. These variables are the axial displacement and the tangential displacement. Figure 4 shows the geometry and standard nomenclature related to tandem airfoils in cascade [12].

The tandem configuration has two key physical effects that are of interest to compressor designers. The first is the circulation effect. Whenever an object, even a blunt one such as a cylinder, is placed downstream of an airfoil, the effect of that object is to increase the circulation around the airfoil. The Kutta- Joukouski law, expressed as, indicates that the lift force is proportional to the circulation. In terms of a tandem compressor rotor, this means that the aft blade will increase the loading on the forward blade, resulting in a greater combined pressure rise between both blades.

$$
F_{L}=\rho U \Gamma
$$

The second effect is the fresh boundary layer that is formed on the aft blade. Ideally, the aft blade would be placed such that it relieves the forward blade just prior to the point of separation, as shown in Figure 4. This allows for greater overall turning of the airflow, hence more work (i.e., pressure rise) while not incurring substantially higher losses.

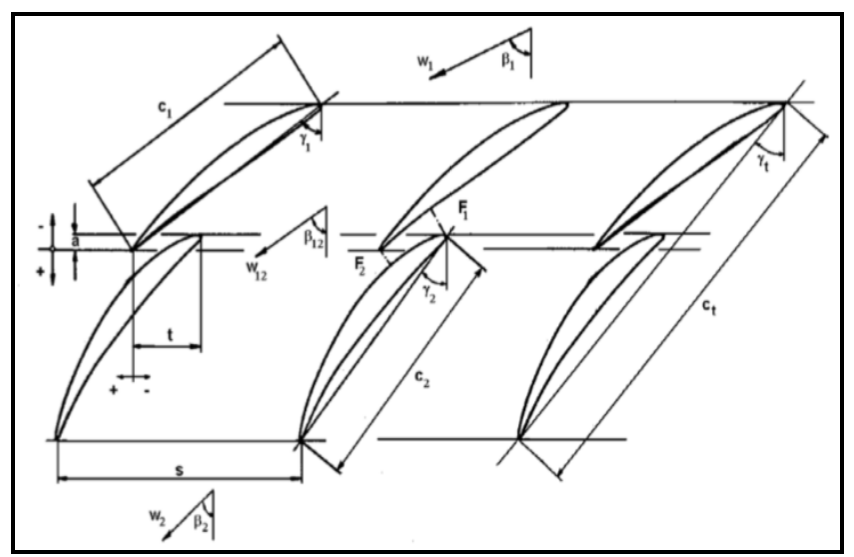

$\begin{array}{llll}\mathrm{a} & \text { Axial displacement } & \mathrm{w}_{12} & \text { Inlet velocity at rear blade } \\ \mathrm{F}_{1} & \text { Inlet gap distance } & \mathrm{w}_{2} & \text { Outlet velocity } \\ \mathrm{F}_{2} & \text { Outlet gap distance } & \beta_{1} & \text { Inlet flow angle } \\ \mathrm{c}_{1} & \text { Chord length front blade } & \beta_{12} & \text { Inlet flow angle at rear blade } \\ \mathrm{c}_{2} & \text { Chord length rear blade } & \beta_{2} & \text { Outlet flow angle } \\ \mathrm{c}_{\mathrm{t}} & \text { Overall chord length } & \gamma_{1} & \text { Stagger angle front blade } \\ \mathrm{s} & \text { Blade spacing } & \gamma_{2} & \text { Stagger angle rear blade } \\ \mathrm{t} & \text { Tangential displacement } & \gamma_{\mathrm{t}} & \text { Overall stagger angle } \\ \mathrm{w}_{\mathrm{l}} & \text { Inlet velocity } & & \end{array}$

Figure 4. Tandem blade nomenclature [12].

\section{Governing Equations}

The incompressible, two-dimensional, potential flow governed by the Laplace equation is solved numerically with a panel method, which provides the tangential external velocity. The pressure is then obtained using the Bernoulli equation, and $C_{l}$ is derived by integrating the pressure over the airfoil.

\subsection{Theoretical Equation of the Flow}

For an irrotational flow, Figure 5, the velocity is the gradient of a quantity called the velocity potential $\phi$.

$$
V=(u, v)=\nabla \phi
$$

Substituting this into the continuity equation for an inviscid incompressible flow leads to:

$$
\frac{\partial^{2} \phi}{\partial x^{2}}+\frac{\partial^{2} \phi}{\partial y^{2}}=0 \text { or } \Delta \phi=0 \text { (Laplace equation) }
$$

On the airfoil's surface "A", Figure 5, the external Neumann boundary condition must be satisfied:

$$
\frac{\partial \phi}{\partial n}=V_{n}
$$

Where, $V_{n}$ is set to zero, which represents the classical zero normal velocity condition.

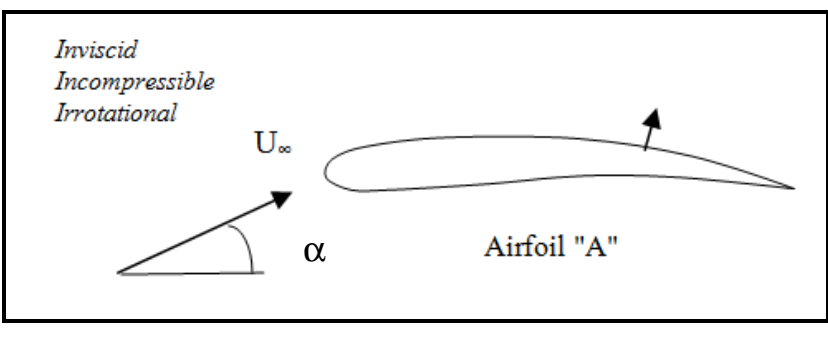

Figure 5. Flow field of an airfoil.

\subsection{General Solution of the Incompressible Potential Flow}

A general solution to the Laplace equation is obtained by adding a distribution of vortices $\gamma$ on the airfoil's surface to the velocity potential of the free-stream, Figure 6 . The solution at any field point $P$ is thus given by [13]:

$$
\phi_{p}=u_{\infty} \cdot x+v_{\infty} \cdot y+\int_{A} \gamma \phi_{v} d s
$$

Where $\phi_{v}$ is the potential of a unit strength vortex: $(r, \theta)$ are 
the polar coordinates of $P$ relative to $(d s)$.

$$
\phi_{v}=-\frac{1}{2 \pi} \theta
$$

This equation has to satisfy the boundary condition for every point on "A", which gives:

$$
\nabla \phi_{p} . n=0 \Rightarrow\left(u_{\infty}, v_{\infty}\right) \cdot n+\int_{A} \gamma \frac{\partial \phi_{v}}{\partial n} d s=0
$$

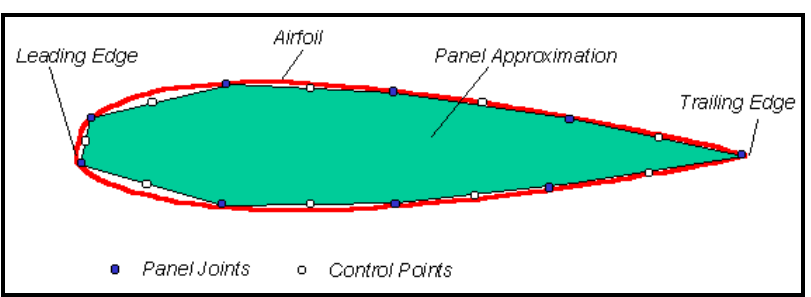

Figure 6. Vortex-panel approximation to an airfoil [13].

Moreover, the integration is performed on each panel and the boundary condition becomes:

$$
\left(u_{\infty}, v_{\infty}\right) \cdot n+\sum_{i=1}^{n}\left(\int_{\text {panel }} \gamma \frac{\partial \phi_{v}}{\partial n} d s\right)=0
$$

\subsection{Singularity Element}

Now, the integral on each panel must be computed:

$$
\int_{\text {panel }} \gamma \frac{\partial \phi_{v}}{\partial n} d s=\left(\int_{\text {panel }} \gamma \frac{\partial \phi_{v}}{\partial x} d s, \int_{\text {panel }} \gamma \frac{\partial \phi_{v}}{\partial y} d s\right) \cdot n \equiv(u, v) . n
$$

We will compute $u, v$ in the panel coordinate system, and then transform them back in the global coordinate system, Figure 7.

Consider the coordinates $(\mathrm{x}, \mathrm{y})$ in the panel system. They are obtained using the following transformation:

$$
\left(\begin{array}{l}
x \\
y
\end{array}\right)_{p}=\left[\begin{array}{cc}
\cos \alpha_{i} & -\sin \alpha_{i} \\
\sin \alpha_{i} & \cos \alpha_{i}
\end{array}\right]\left(\begin{array}{l}
x-x_{0} \\
y-y_{0}
\end{array}\right)_{G}
$$

Where, $\left(x_{0}, y_{0}\right)$ are the coordinates of the panel origin in the global coordinate system.

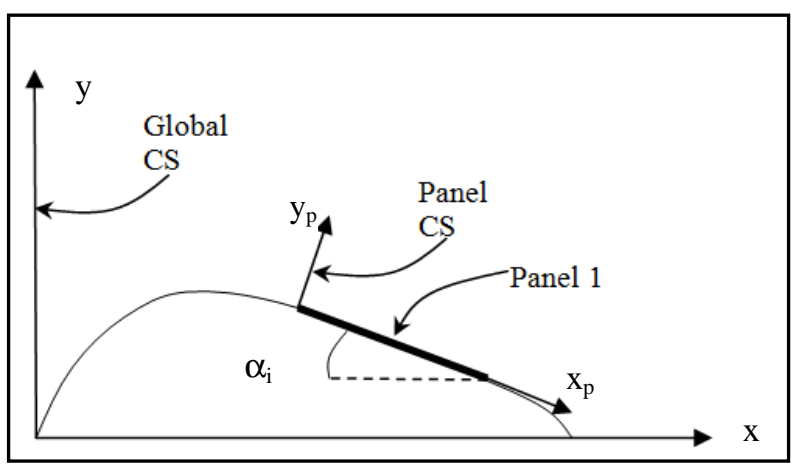

Figure 7. Global and panel coordinate systems.

On each panel, we choose a linear vortex distribution,
Figure 8, such as

$$
\gamma(x)=\gamma_{0}+\gamma_{1} x
$$

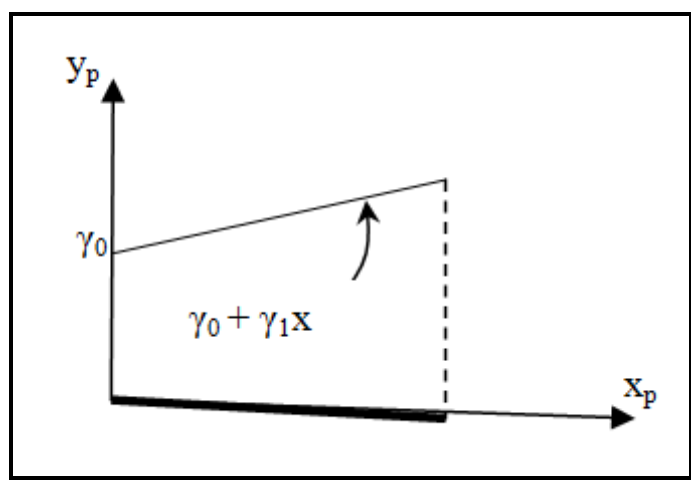

Figure 8. Linear vortex distribution of a panel.

This is simply the superposition of a constant strength element and a linearly varying element. For simplicity, we consider the two elements separately to compute the potential and the velocity induced by one panel.

$$
\begin{gathered}
\phi_{\gamma_{0}}=\frac{-\gamma_{0}}{2 \pi} \int_{0}^{L} a t \tan \frac{y}{x-x_{0}} d x_{0} \\
\text { so: } u_{\gamma_{0}}=\frac{\partial \phi_{\gamma_{0}}}{\partial x}=\frac{\gamma_{0}}{2 \pi} \int_{0}^{L} \frac{y}{\left(x-x_{0}\right)^{2}+y^{2}} d x_{0} \\
\text { and: } u_{\gamma_{0}}=\frac{\partial \phi_{\gamma_{0}}}{\partial y}=\frac{\gamma_{0}}{2 \pi} \int \frac{x-x_{0}}{\left(x-x_{0}\right)^{2}+y^{2}} d x_{0}
\end{gathered}
$$

Integrating the above two equations we obtain:

$$
u_{\gamma_{0}}=\frac{\gamma_{0}}{2 \pi}\left(\theta_{2}-\theta_{1}\right) \text { and } v_{\gamma_{0}}=\frac{\gamma_{0}}{2 \pi} \ln \frac{r_{2}}{r_{1}}
$$

We consider the linear term now:

$$
\begin{gathered}
\phi_{\gamma 1 x}=\frac{\partial \phi_{\gamma 1 x}}{\partial x} \int_{0}^{L} x_{0} a \tan \frac{y}{x-x_{0}} d x_{0} \\
\text { so: } u_{\gamma 1 x}=\frac{\partial \phi_{\gamma 1 x}}{\partial x}=\frac{\gamma_{1}}{2 \pi} \int_{0}^{L} \frac{x_{0} y}{\left(x-x_{0}\right)^{2}+y^{2}} d x_{0} \\
\text { and: } v_{\gamma 1 x}=\frac{\partial \phi_{\gamma 1 x}}{\partial y}=\frac{\gamma_{1}}{2 \pi} \int_{0}^{L} \frac{x_{0}\left(x-x_{0}\right)}{\left(x-x_{0}\right)^{2}+y^{2}} d x_{0}
\end{gathered}
$$

Solving the integrals gives:

$$
\begin{gathered}
u_{\gamma 1 x}=-\frac{\gamma_{1}}{4 \pi}\left[2 z \ln \frac{r_{1}}{r_{2}}-2 x\left(\theta_{2}-\theta_{1}\right)\right] \\
v_{\gamma 1 x}=-\frac{\gamma_{1}}{2 \pi}\left[x \ln \frac{r_{1}}{r_{2}}+L+z\left(\theta_{2}-\theta_{1}\right)\right]
\end{gathered}
$$

Now, what we want is a piecewise linear continuous vortex distribution on the whole airfoil surface. So, we have to set the strength $\gamma$ at the beginning of each panel equal to the strength of the vortex at the end of the previous panel as shown in 
Figure 9.

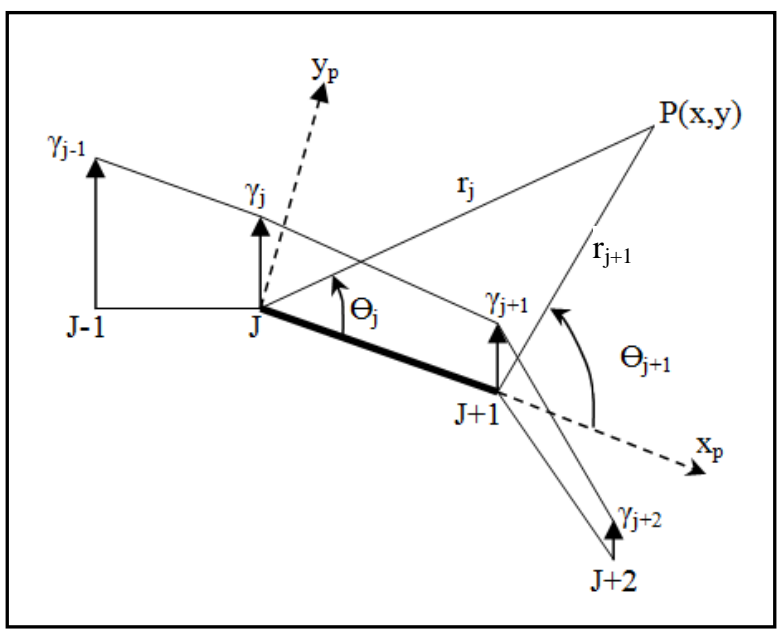

Figure 9. Piecewise linear continuous vortex distribution.

The relations between the vortex strengths of the elements shown in Figure 9 and the panel end values $\gamma_{0}$ and $\gamma_{l}$ are:

$$
\gamma_{j}=\gamma_{0} \text { and } \gamma_{j+1}=\gamma_{0}+\gamma_{1} L
$$

Thus, rearranging the expressions for $u$ and $v$ in terms of $\gamma_{j}$ and $\gamma_{j+1}$ gives:

$$
\begin{aligned}
& u=\frac{z}{2 \pi}\left(\frac{\gamma_{j+1}-\gamma_{j}}{x_{j+1}-x_{j}}\right) \ln \frac{r_{j+1}}{r_{j}}+ \\
& \frac{\gamma_{j}\left(x_{j+1}-x_{j}\right)+\left(\gamma_{j+1}-\gamma_{j}\right)\left(x-x_{j}\right)}{2 \pi\left(x_{j+1}-x_{j}\right)}\left(\theta_{j+1}-\theta_{j}\right) \\
& v=\frac{\gamma_{j}\left(x_{j+1}-x_{j}\right)+\left(\gamma_{j+1}-\gamma_{j}\right)\left(x-x_{j}\right)}{2 \pi\left(x_{j+1}-x_{j}\right)} \ln \frac{r_{j+1}}{r_{j}} \\
& +\frac{y}{2 \pi}\left(\frac{\gamma_{j+1}-\gamma_{j}}{x_{j+1}-x_{j}}\right)\left[\left(\frac{x_{j+1}-x_{j}}{z}\right)+\left(\theta_{j+1}-\theta_{j}\right)\right]
\end{aligned}
$$

These two equations can be divided into velocity induced by $\gamma_{j+1}$ and $\gamma_{j}$ such that:

$$
(u, v)=\left(u^{a}, v^{a}\right)+\left(u^{b}, v^{b}\right)
$$

Where, the superscripts " $a$ " and " $b$ " represent the contribution due to the leading and trailing singularity. By rearranging the equations, we obtain the " $a$ " part of the velocity:

$$
\begin{aligned}
& u^{a}=\frac{\gamma_{j}}{2 \pi\left(x_{j+1}-x_{j}\right)}\left[-z \ln \frac{r_{j+1}}{r_{j}}\right]+\left(x_{j+1}-x\right)\left(\theta_{j+1}-\theta_{j}\right) \\
& v^{a}=\frac{-\gamma_{j}}{2 \pi\left(x_{j+1}-x_{j}\right)}\left[\left(x_{j+1}-x_{j}\right) \ln \frac{r_{j}}{r_{j+1}}+\left(x_{j+1}-x_{j}\right)+z\left(\theta_{j+1}-\theta_{j}\right)\right]
\end{aligned}
$$

and the " $b$ " part of the velocity:

$$
u^{b}=\frac{\gamma_{j+1}}{2 \pi\left(x_{j+1}-x_{j}\right)}\left[z \ln \frac{r_{j+1}}{r_{j}}\right]+\left(x-x_{j}\right)\left(\theta_{j+1}-\theta_{j}\right)
$$

$$
\left.v^{b}=\frac{\gamma_{j+1}}{2 \pi\left(x_{j+1}-x_{j}\right.}\right)\left[-\left(x-x_{j}\right) \ln \frac{r_{j}}{r_{j+1}}+\left(x_{j+1}-x_{j}\right)+z\left(\theta_{j+1}-\theta_{j}\right)\right]
$$

To transform these velocity components back to the global coordinate system, a rotation by the panel orientation angle $\alpha_{i}$ is performed as given by:

$$
\left(\begin{array}{l}
u \\
v
\end{array}\right)_{G}=\left[\begin{array}{cc}
\cos \alpha_{i} & \sin \alpha_{i} \\
-\sin \alpha_{i} & \cos \alpha_{i}
\end{array}\right]\left(\begin{array}{l}
u \\
w
\end{array}\right)_{P}
$$

The expressions above can be included in an induced velocity function $F$, which will compute the velocity $(u, v)$ at an arbitrary point $(x, y)$ in the global coordinate system due to the $j$-th panel.

$$
\left(\begin{array}{cc}
u^{a} & v^{a} \\
u^{b} & v^{b}
\end{array}\right)=F\left(\gamma_{j}, \gamma_{j+1}, x, z, x_{j}, y_{j}, x_{j+1}, y_{j+1}\right)
$$

\subsection{Influence Coefficients}

The zero normal flow boundary condition is implemented. For example the velocity induced by the $j$-th element with unit strength at the first collocation point is obtained by:

$$
\left(\begin{array}{ll}
u^{a} & v^{a} \\
u^{b} & v^{b}
\end{array}\right)_{1 j}=F\left(\gamma_{j}=1, \gamma_{j+1}=1, x_{1}, z_{1}, x_{j}, y_{j}, x_{j+1}, y_{j+1}\right)
$$

This shows that the velocity at each collocation point is influenced by the two edges of the $j$-th panel, When adding the influence of the $j+1$ panel on the local induced velocity will have the form:

$$
\begin{aligned}
& (u, v)_{1}=\left(u^{a}, v^{a}\right)_{11} \gamma_{1}+\left\lfloor\left(u^{b}, v^{b}\right)_{11}+\left(u^{a}, v^{a}\right)_{12} \gamma_{2}+\right. \\
& \ldots .+\left[\left(u^{b}, v^{b}\right)_{1 N-1}+\left(u^{a}, v^{a}\right)_{1 N}\right] \gamma_{N}+\left(u^{b}, v^{b}\right)_{1 N} \gamma_{N+1}
\end{aligned}
$$

This equation can be reduced to the form:

$$
(u, v)_{1}=(u, v)_{11} \gamma_{1}+(u, v)_{12} \gamma_{2}+\ldots .+(u, v)_{1 N} \gamma_{N}+(u, v)_{1 N+1} \gamma_{N+1}
$$

Such that for the first and last terms:

$$
(u, v)_{11}=\left(u^{a}, v^{a}\right)_{11} \text { and }(u, v)_{1 N+1}=\left(u^{b}, v^{b}\right)_{1 N}
$$

and for all other terms:

$$
(u, v)_{1 j}=\left[\left(u^{b}, v^{b}\right)_{1 j-1}+\left(u^{a}, v^{a}\right)_{1 j}\right]
$$

The influence coefficient $a_{i j}$ is defined as the velocity component normal to the surface. As see in Figure 10, the contribution of a unit strength singularity element $j$ at collection point 1 is therefore:

$$
a_{1 j}=(u, v)_{1 j} \cdot n_{1}
$$

Where: $n_{i}=\left(\sin \alpha_{i}, \cos \alpha_{i}\right)$ 


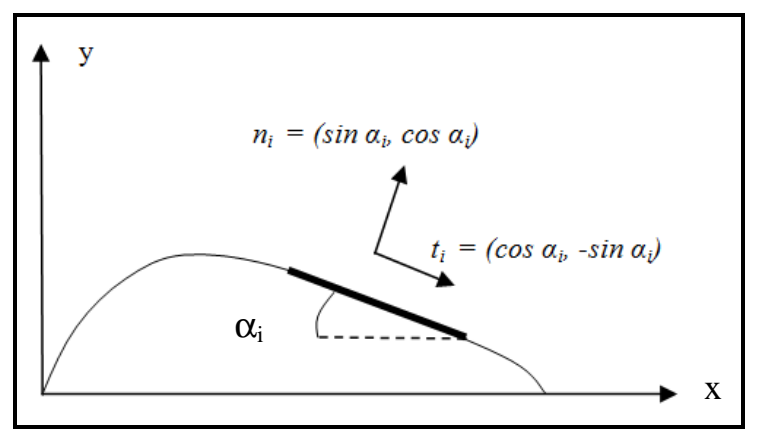

Figure 10. Influence coefficient.

\subsection{Establishing Boundary Conditions}

The free stream normal velocity component is found as:

$$
\operatorname{RHS}_{i}=-\left(u_{\infty}, v_{\infty}\right) \cdot\left(\sin \alpha_{i}, \cos \alpha_{i}\right)
$$

Specifying the boundary condition equation for each $(i=1$ to $N$ ) of the collocation points results in $N$ linear equations with the unknowns $\gamma_{j}(j=1$ to $N+1)$

$$
\left[\begin{array}{cccc}
a_{11} & a_{12} & \cdots & a_{1 N+1} \\
a_{21} & a_{22} & \cdots & a_{2 N+1} \\
\cdots & \cdots & \cdots & \cdots \\
a_{N 1} & a_{N 2} & \cdots & a_{N N+1}
\end{array}\right]\left(\begin{array}{c}
\gamma_{1} \\
\gamma_{2} \\
\cdots \\
\gamma_{N+1}
\end{array}\right)=\left(\begin{array}{c}
R H S_{1} \\
R H S_{2} \\
\cdots \\
R H S_{3}
\end{array}\right)
$$

An additional condition must be established in order to obtain a unique solution that is Kutta condition, which specifies that the circulation at the trailing edge must be zero: $\gamma_{T E}=0$.

For our model, the circulation is given by: $\gamma_{T E}=\gamma_{1}+\gamma_{N+1}$, and the Kutta condition is

$$
\gamma_{1}+\gamma_{N+1}=0
$$

This extra condition is added to the system of equations to give:

$$
\left[\begin{array}{cccc}
a_{11} & a_{12} & \cdots & a_{1 N+1} \\
a_{21} & a_{22} & \cdots & a_{2 N+1} \\
\cdots & \cdots & \cdots & \cdots \\
a_{N 1} & a_{N 2} & \cdots & a_{N N+1} \\
1 & 0 & \cdots & 1
\end{array}\right]\left(\begin{array}{c}
\gamma_{1} \\
\gamma_{2} \\
\cdots \\
\gamma_{N+1}
\end{array}\right)=\left(\begin{array}{c}
R H S_{1} \\
R H S_{2} \\
\cdots \\
R H S_{n} \\
0
\end{array}\right)
$$

The above set of equations can be solved for $\gamma_{i}$ by using standard methods of linear algebra.

\subsection{Calculation of the Velocity}

The velocity is obtained by adding the tangential components of $(u, v)$ of each panel to the tangential component of the external flow velocity.

So, we have to build the $N \times N+1$ matrix $b$ of coefficients $b_{i j}$ such that: $b_{i j}=(u, v)_{i j} \cdot t_{i}$

Where: $t_{i}=\left(\cos \alpha_{i},-\sin \alpha_{i}\right)$ and the vector $\mathrm{Ue}_{\infty}$ of terms: $U e_{\infty}=-\left(u_{\infty}, v_{\infty}\right) \cdot\left(\cos \alpha_{i},-\sin \alpha_{i}\right)$

Then, we have

$$
\left(\begin{array}{c}
U_{e 1} \\
U_{e 2} \\
\cdots \\
U_{e 4}
\end{array}\right)=\left[\begin{array}{cccc}
b_{11} & b_{12} & \cdots & b_{1 N+1} \\
b_{21} & b_{22} & \cdots & b_{2 N+1} \\
\cdots & \cdots & \cdots & \cdots \\
b_{N 1} & b_{N 2} & \cdots & b_{N N+1}
\end{array}\right]\left(\begin{array}{c}
\gamma_{1} \\
\gamma_{2} \\
\cdots \\
\gamma_{N+1}
\end{array}\right)+\left(\begin{array}{c}
U_{e \infty 1} \\
U_{e \infty 2} \\
\cdots \\
U_{e \infty 4}
\end{array}\right)
$$

which gives the tangential velocity at each airfoil collocation point.

\subsection{Computation of the Pressure}

The Bernoulli equation applied to a streamline between the upstream infinity and a point on the airfoil's surface gives:

$$
\begin{array}{r}
p+\frac{1}{2} \rho_{u_{e}}^{2}=p_{\infty}+\frac{1}{2} \rho_{\infty} u_{\infty}^{2} \\
\text { so: } p=p_{\infty}+\frac{1}{2} \rho_{\infty} u_{\infty}^{2}-\frac{1}{2} \rho_{u_{e}^{2}}^{2} \\
\text { then: } C_{P}=\frac{P-p_{\infty}}{0.5 \rho_{\infty} U_{\infty}^{2}}=1-\frac{U_{e}^{2}}{U_{\infty}^{2}}
\end{array}
$$

We can thus compute the pressure coefficient at each airfoil collocation point.

\subsection{Computation of the Aerodynamic Coefficients}

As shown in Figure 11, the elementary forces $f_{x j}$ and $f_{y j}$ acting on panel $j$ is obtained as:

$$
f_{x j}=c p_{j}\left(y_{j+1}-y_{j}\right) \text { and } f_{y j}=c p_{j}\left(x_{j+1}-x_{j}\right)
$$

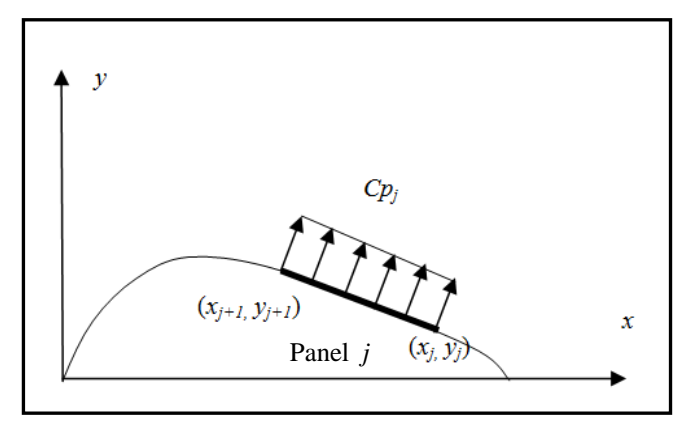

Figure 11. Elementary forces on panel $j$.

By doing this for each panel and by adding the elementary forces the total load applied to the airfoil is obtained $\left(F_{x}, F_{y}\right)$.

The lift coefficient is then the component of $F$ normal to the flow direction:

$$
C_{L}=-\sin \alpha \cdot F_{x}+\cos \alpha \cdot F_{y}
$$

\section{Computational Code and Test Cases}

The flow along the tandem blade will be studied using different simulations for various relative blade positions. In all the tandem models, the front blade is fixed and the rear blade varies its position as a function of the axial and tangential displacements. 
Two different approaches are chosen to identify the influence of location of the rear blade in the tandem arrangement:

- The blade is positioned in such a way that there are no gap nozzle effects between the blades.

- The blade is positioned in the vicinity of the trailing edge of the front blade in order to create a gap nozzle area.

A "Visual Basic" code was developed using linear strength vortex-panel method to predict the aerodynamic performance of tandem blades for various relative blade positions, Figure 12.

\subsection{Initial Configuration}

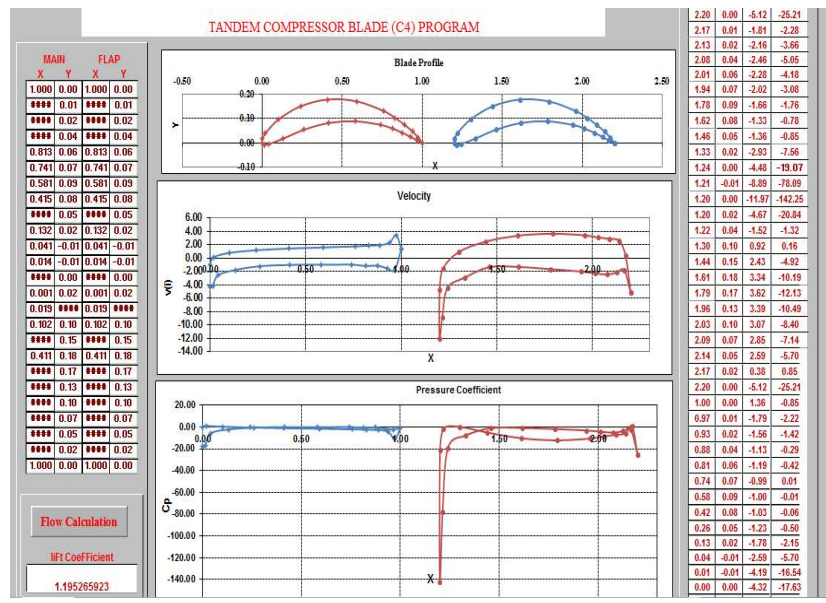

Figure 12. Tandem blade program profile.

The first step in the investigation is to characterize the flow when the second blade of the tandem blades is positioned in the "no gap nozzle effect", Figure 13. The interaction between the two blades is only evident in terms of the wake of the front blade that affects the flow behavior along the arrangement. To characterize the influence of the second blade position, different values are given for the axial and tangential displacements of the second blade. Figure 14 shows a graphical representation of the front blade's trailing edge with the possible second blade locations in terms of the axial and tangential displacements. There are a range of three axial displacements that varies between 0 and 0.2 and a range of five tangential displacements that varies between 0.15 and -0.15 . These two ranges represent relative position of the rear blade with respect to the front blade. All the displacements (axial/tangential) are measured from the trailing edge of the front blade.

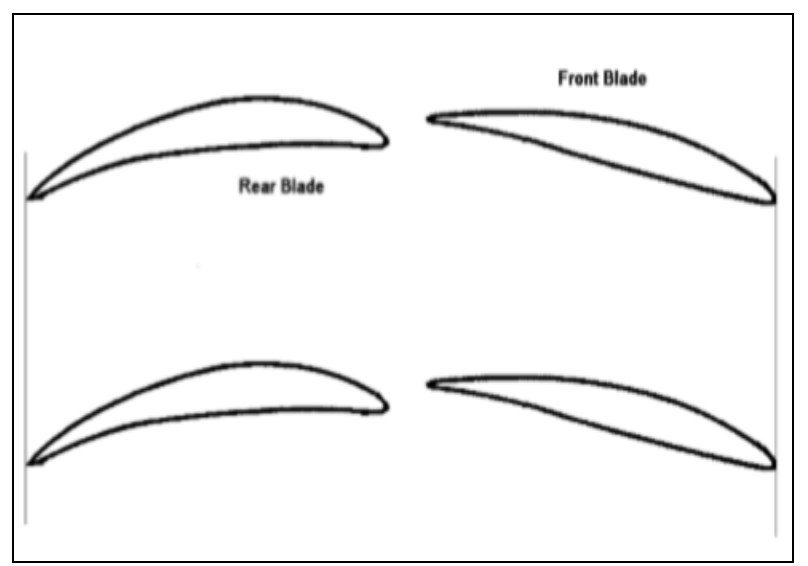

Figure 13. Tandem blade arrangement with "no gap nozzle area" between the profiles [7].

Figures 15-19 show the influence of the rear blade position on the pressure coefficient and velocity distributions. The axial displacement for all figures is set to $20 \%$ of the chord of the front blade. Table 3 illustrates the relative positions of the tandem blades for the case of "no gap nozzle effect".

\subsection{Inlet Conditions}

The incoming flow has a free-stream velocity of $1 \mathrm{~m} / \mathrm{s}$, an angle of attack of $8^{\circ}$ and an inlet flow angel of $35^{\circ}$.

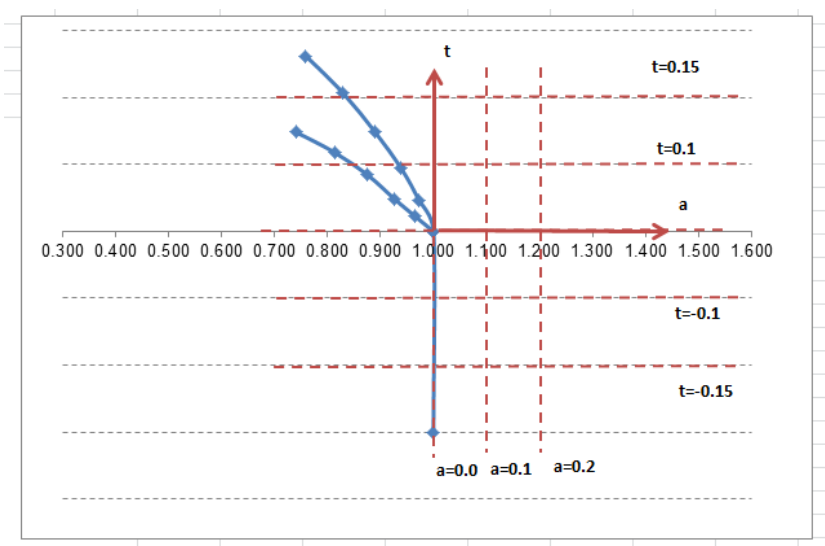

Figure 14. Terminology of relative positions of tandem blades.

Table 3. Relative positions of the tandem blades

\begin{tabular}{lll}
\hline Axial displacement $[\boldsymbol{a}]$ & Tangential displacement $[\boldsymbol{t}]$ & Comments \\
\hline 0.2 & 0.0 & The second blade is positioned in the viscous-free region of the first blade \\
0.2 & 0.1 & "No gap nozzle effect" between the two blades \\
0.2 & 0.15 & "No gap nozzle effect" between the two blades \\
0.2 & -0.1 & "No gap nozzle effect" between the two blades \\
0.2 & -0.15 & "No gap nozzle effect" between the two blades \\
\hline
\end{tabular}

\section{Results and Discussions}

\subsection{Original Blade Configuration}

Figure 15a shows the relative positions of tandem blades for $\mathrm{a}=0.2, \mathrm{t}=0$. Figure $15 \mathrm{~b}$ demonstrates the velocity distribution of the tandem blades when the second blade is positioned in the viscous-free region of the front blade. The velocity distribution for the forward blade shows a steady constant acceleration on the suction surface over most of the 
chord length. On the pressure surface, the velocity drops to a low value (-4) and then increases up till $x / C=0.3$. Then, for $0.3<x / C<0.97$, velocity is almost constant. A small sudden drop of velocity is found at $x / C=0.97$, followed by a rise in velocity toward trailing edge.

For the rear blade, on the suction surface, the velocity drops to a low value (-12) followed by a steady rise up to $x / C=0.97$. Then, a sudden drop occurs toward the trailing edge. On the pressure surface, the velocity is almost constant over most of the chord. Then, velocity drops suddenly towards the trailing edge. This is a consequence of the interaction between the wake of the front blade and the velocity field in the vicinity of the suction surface of the rear blade.

For the front blade, Figure 15c shows almost constant pressure on the suction surface due to the steady constant acceleration mentioned previously in Figure 15b. Similar behavior is noticed on the pressure surface except at the leading and trailing edges.

For the rear blade, on the suction surface, the pressure diffuses steadily till $x / C=0.1$. Then, pressure drops in the range $0.1<x / C<0.6$ followed by a steady rise in the range $0.6<$ $x / C<0.9$. Finally, the flow separates at the trailing edge due to the low flow-momentum produced by the low velocity in that region as shown in Figure 15b. On the pressure surface, the pressure is almost constant for most of the chord.

Figures 16-19 illustrate the velocity and pressure distributions of the tandem blades when the rear blade is positioned at $(a=0.2, t=0.1),(a=0.2, t=0.15),(a=0.2, t=-0.1)$ and $(a=0.2, t=-0.15)$. Generally, there is almost no difference in pressure and velocity distributions with the first case, when the rear blade is placed at the wake of the front blade $(a=0.2, t$ $=0.0$ ).

In Figures 15-19, it is clear that the flow behavior along the suction surface of the front blade is not affected by the relative position of the rear blade. This may be explained as a consequence of the no-interaction between the blades. However, there is a noticeable variation of the maximum negative pressure at the leading edge of the rear blade. This may be attributed to a higher inlet velocity to the rear blade due to its relative axial position.

The negative value of $C_{p}$ for $t=-0.15$ is smaller than that of $t=-0.1$. Similar behavior is noticed for the two cases of $t=0.1$ and $t=0.15$.

It was not possible to obtain results for the tandem blades with $a>0.2$ and $t>0.15$ because the model did not converge due to massive flow separation on the suction surface of the rear blade.

Figure 20 illustrates the lift coefficient of the rear blade at fixed axial displacement $(a=0.2)$ and different tangential displacements $(t)$.

The lift coefficient increases as the rear blade gets away from the front blade. This may be attributed to the weak interaction between the two blades. Maximum value of lift coefficient is recorded at $t=-0.1$.

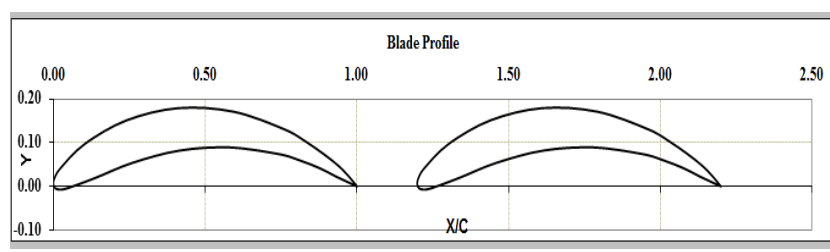

Figure 15a. Relative positions of tandem blades, $a=0.2, t=0$.

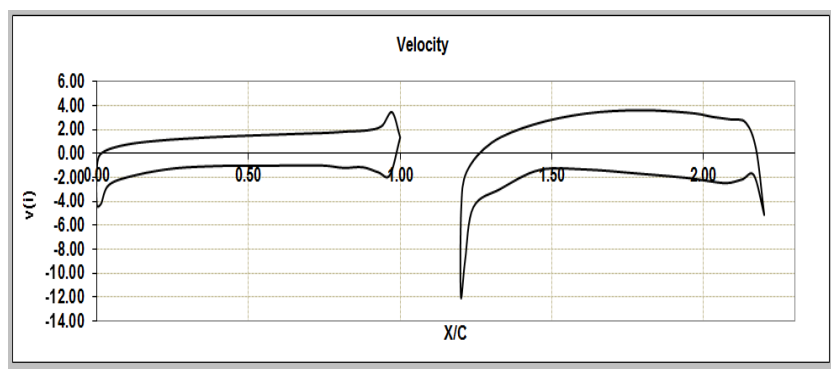

Figure 15b. Velocity distributions, $a=0.2, t=0$

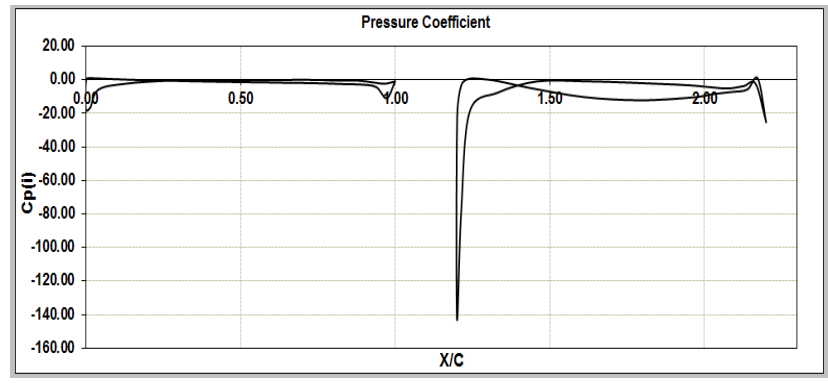

Figure 15c. Distributions of pressure coefficient, $a=0.2, t=0$.

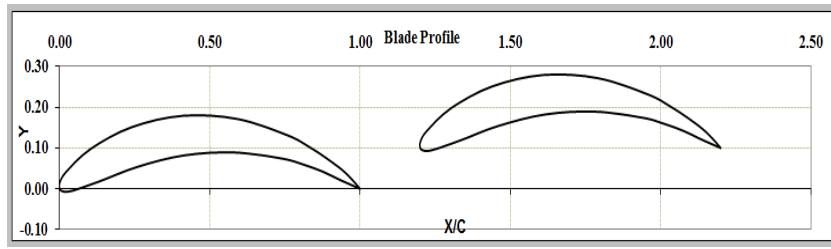

Figure 16a. Relative positions of tandem blades, $a=0.2, t=0.1$.

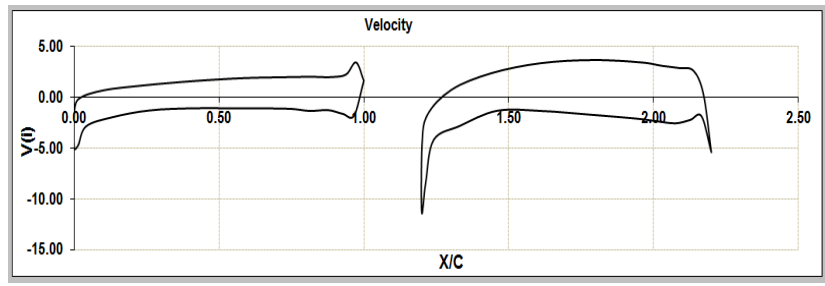

Figure 16b. Velocity distributions, $a=0.2, t=0.1$.

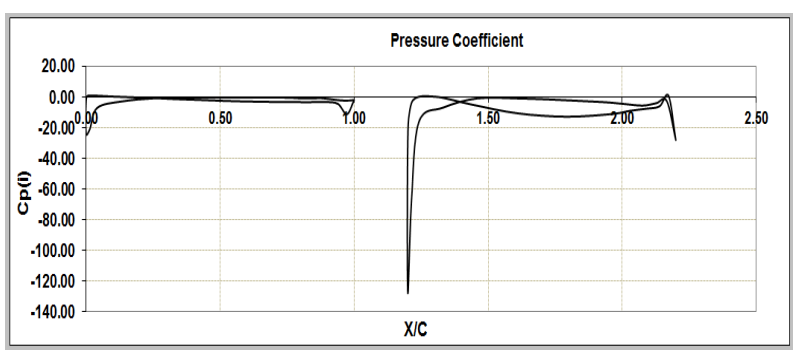

Figure 16c. Distributions of pressure coefficient, $a=0.2, t=0.1$. 


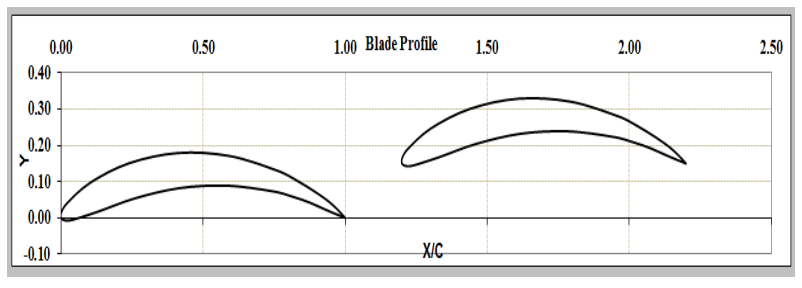

Figure 17a. Relative positions of tandem blades, $a=0.2, t=0.15$.

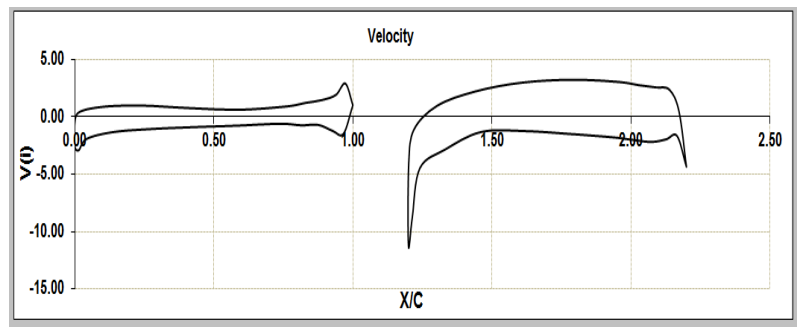

Figure 17b. Velocity distributions, $a=0.2, t=0.15$.

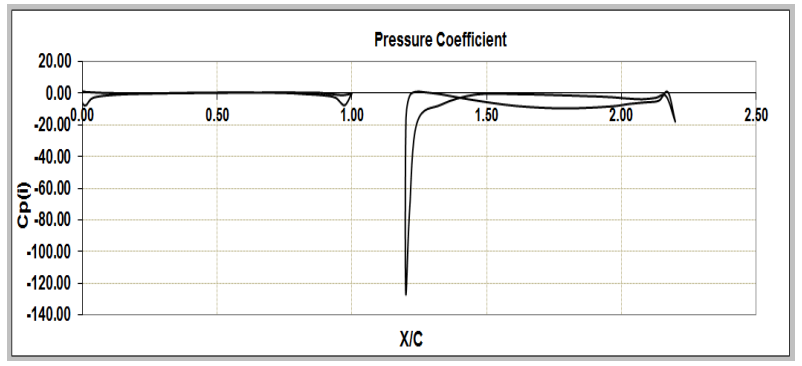

Figure 17c. Distributions of pressure coefficient, $a=0.2, t=-0.1$.

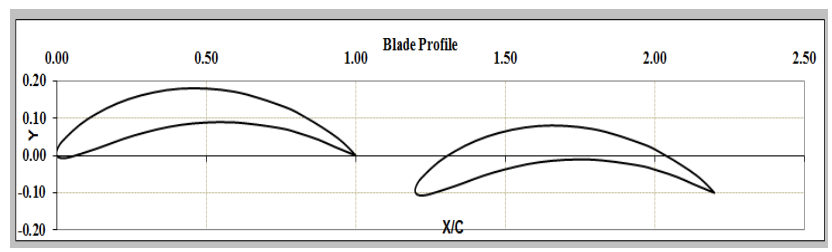

Figure 18a. Relative positions of tandem blades, $a=0.2, t=-0.1$.

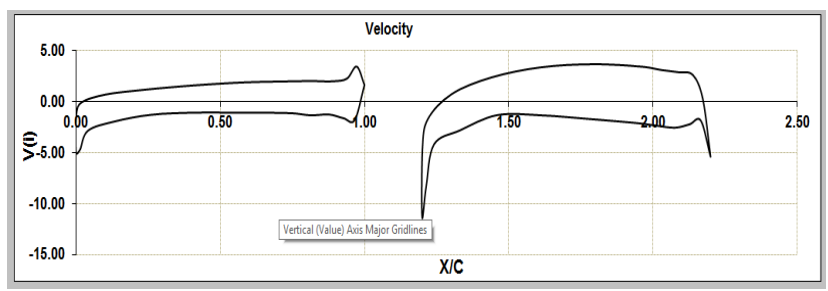

Figure 18b. Velocity distributions, $a=0.2, t=-0.1$.

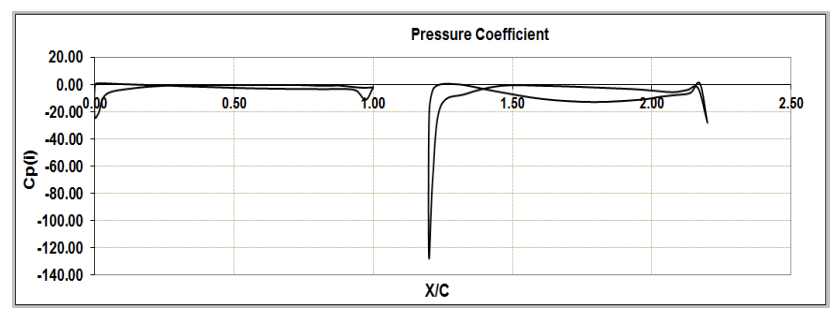

Figure 18c. Distributions of pressure coefficient, $a=0.2, t=-0.1$.

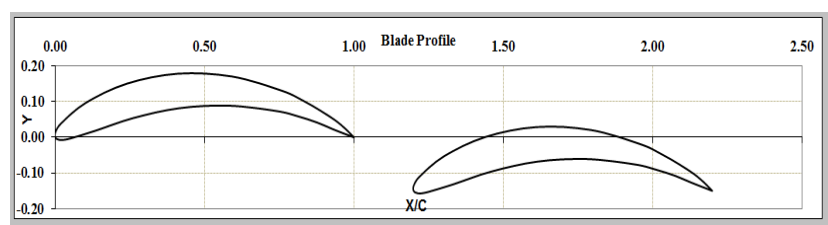

Figure 19a. Relative positions of tandem blades, $a=0.2, t=-0.15$.

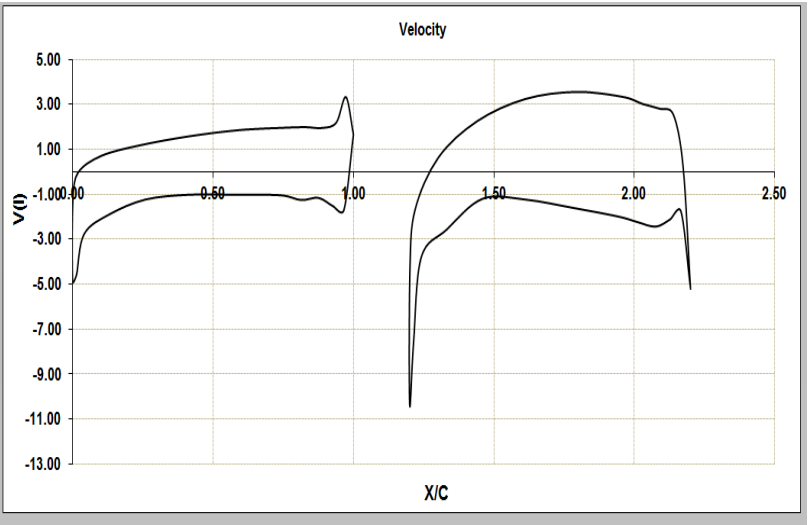

Figure 19b. Velocity distributions, $a=0.2, t=-0.15$.

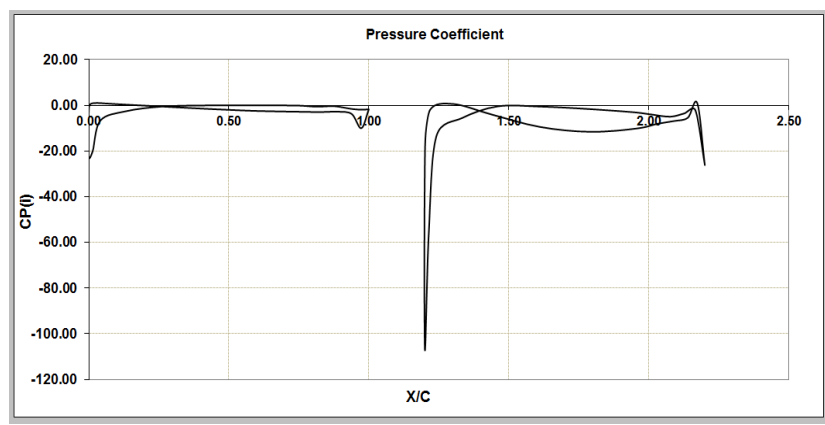

Figure 19c. Distributions of pressure coefficient, $a=0.2, t=-0.15$.

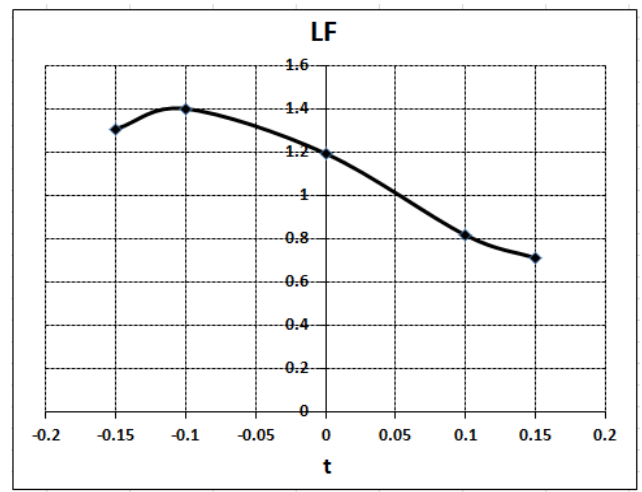

Figure 20. Lift coefficient of rear blade at fixed axial displacement $a=0.2$ and different tangential displacements $(t)$.

As a collection of the results that were shown in Figures 15-19, Figures $21 \mathrm{a}$ and $21 \mathrm{~b}$ illustrates the velocity distributions on the front and rear blades, respectively. Generally, it is clear from Figure 21a that the flow along the pressure surface of the front profile is slightly decelerated in comparison to the case of tangential displacement $(t=0)$. However, the velocity on the suction surface varies considerably depending on the relative location of the rear 
blade. Figure $21 \mathrm{~b}$ demonstrates that that there is a very limited effect of the relative location of the rear blade on the velocity distributions on both the suction and pressure surfaces of the rear blade.

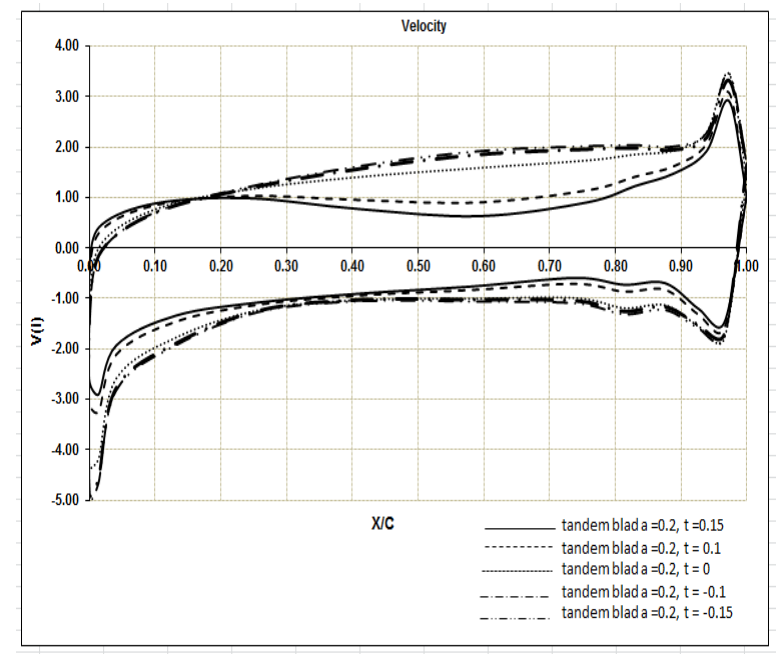

Figure 21a. Velocity distribution on front blade, "No gap nozzle effect".

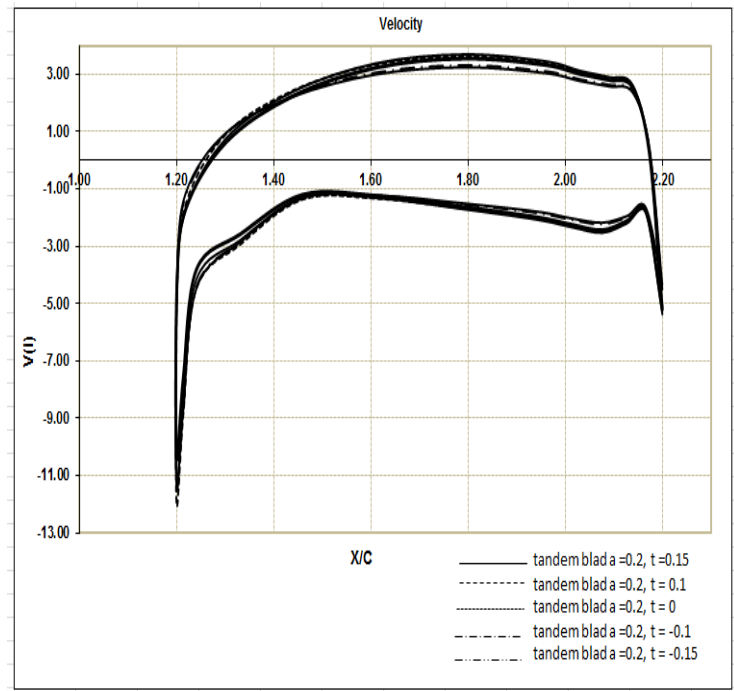

Figure 21b. Velocity distribution on rear blade, "No gap nozzle effect".

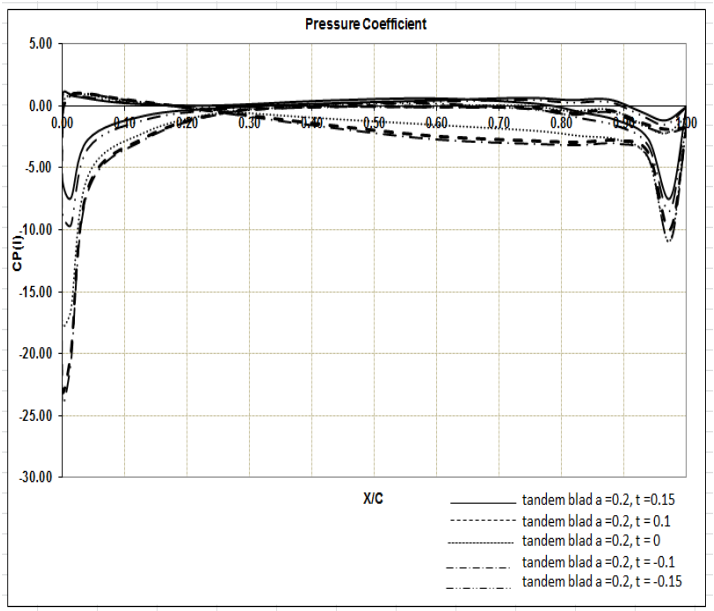

Figure 22a. Pressure distribution on front blade, "No gap nozzle effect".
Figure 22a shows the pressure distribution on the front blade. On the pressure surface, there is a big increase of the negative value of the pressure near the leading edge due to the changes of velocity in this region (Figure 21a). On the suction surface, the pressure values increase or decrease depending on the changes of velocity (Figure 21a) according to the relative position of the rear blade.

Again, Figure 22b shows minor effect of the relative position of the rear blade on the pressure distributions on both the suction and pressure surfaces (similar to Figure 21b).

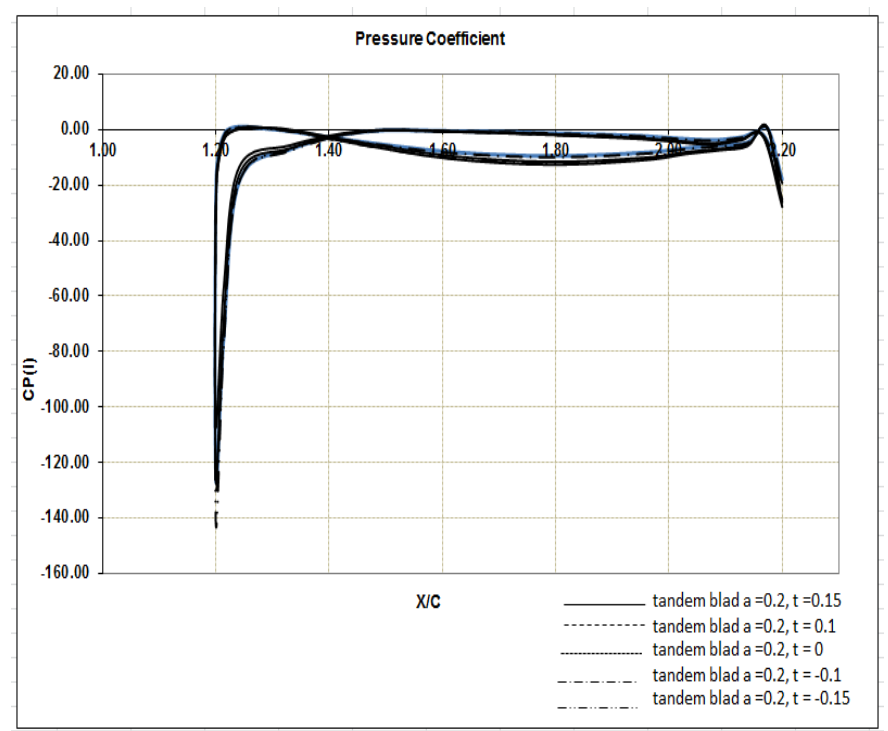

Figure 22b. Pressure distribution on rear blade, "No gap nozzle effect".

\subsection{Variable Camber Blade}

The concept of changing the camber of the rear blade is usually used to vary only the blade stagger of the stator rows in case of "no gap nozzle effect", Figure 23. The versatility of the tandem (rear) blade lies on its variable loading capability. This may be achieved by changing the stagger of the rear blade only; and thereby increasing the overall camber of the blades.

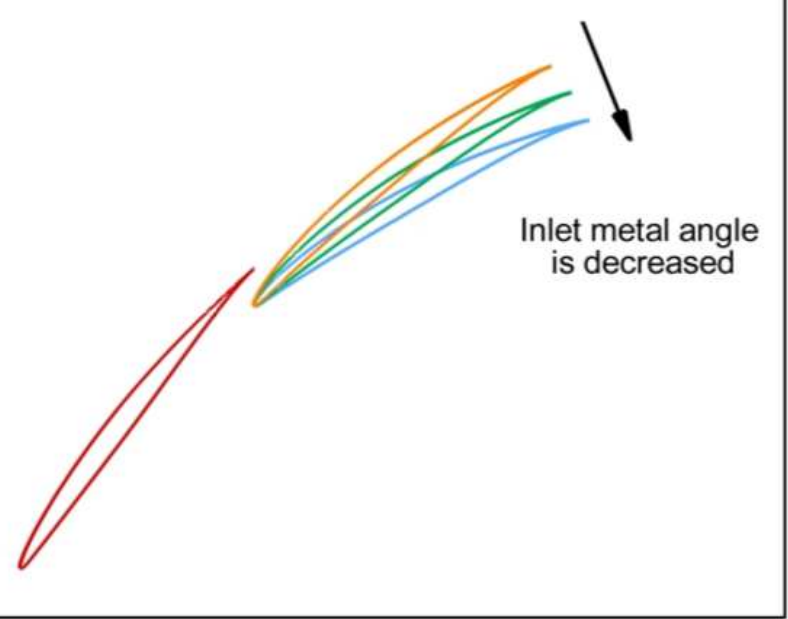

Figure 23. Increasing overall camber of the two tandem blades. 
Several test cases were considered to demonstrate the increase in overall camber of the tandem blades by changing the stagger of the rear blade in the range from $10^{\circ}$ to $60^{\circ}$ relative to the front blade.

Figure 24a demonstrates the case of the stagger angle of the rear blade equals to $10^{\circ}$ with relative position $a=0.2, t=-0.1$ in respect to the front blade. The velocity distributions, Figure $24 \mathrm{~b}$, show an increase in velocity levels on both the front and rear blades as compared to the corresponding case with no change in the stagger angle of the rear blade (Figure 18b). The increase in the velocity level of the front blade is probably due to the increase of the loading of the front blade due to the change in the stagger angle of the rear blade. While the increase in the velocity level of the rear blade is due to better inlet flow angle to the rear blade, i.e., good flow path over the suction and pressure surfaces of the rear blade. As for the pressure distributions of the front and rear blades, Figure $24 \mathrm{c}$,there is a noticeable increase in the pressure values especially near the leading edge of the rear blade in comparison to the corresponding case with no change in the stagger angle of the rear blade (Figure 18c). This may be attributed to velocity changes (Figure 24b).

Figures 25-30 illustrate the change of the velocity and pressure distributions for different stagger angles $\left(15^{\circ}, 20^{\circ}\right.$, $30^{\circ}, 40^{\circ}, 50^{\circ}, 60^{\circ}$ ) of the rear blade relative to the front blade.

Figure 31 shows the variation of the lift coefficient of the rear blade with the stagger angle. It is clear that the lift coefficient increases with the increase of stagger angle due to increase in flow attachment to the surface of the blade till $50^{\circ}$, where it reaches a maximum value of 2.312 . Then, the lift coefficient starts to decrease due to flow separation. Hence, the increase of camber becomes less beneficial. This value of 2.312 is a considerable increase of the lift coefficient when compared to the original configuration, Figure 20, where the maximum value was 1.4 .

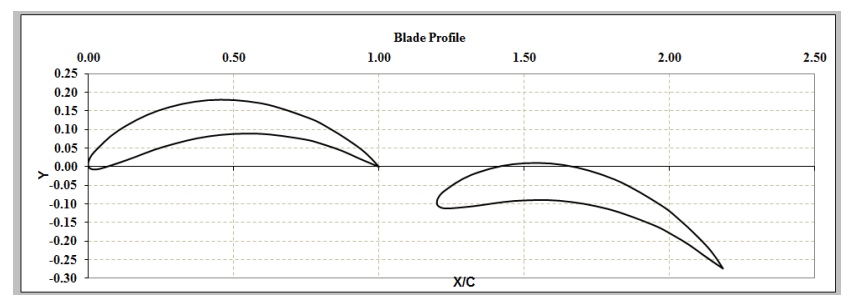

Figure 24a. Relative positions of tandem blades, $a=0.2, t=-0.1$, rear blade stagger angle $\lambda=10^{\circ}$

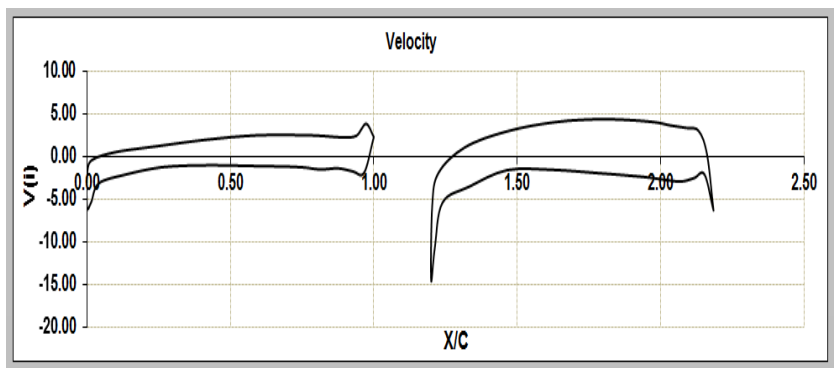

Figure 24b. Velocity distributions, $a=0.2, t=-0.1$, rear blade stagger angle $\lambda=10^{\circ}$.

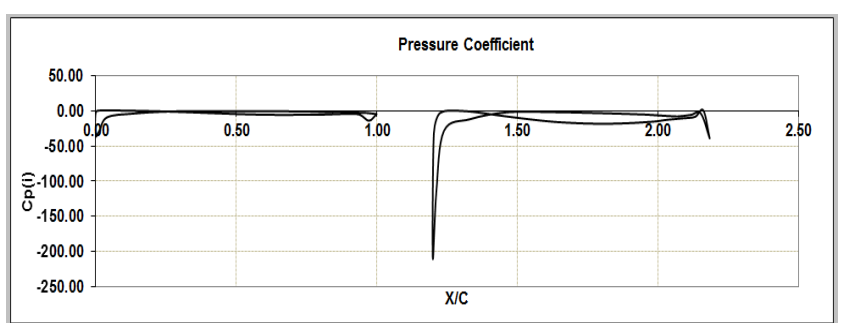

Figure 24c. Distributions of pressure coefficient, $a=0.2, t=-0.1$, rear blade stagger angle $\lambda=10^{\circ}$.

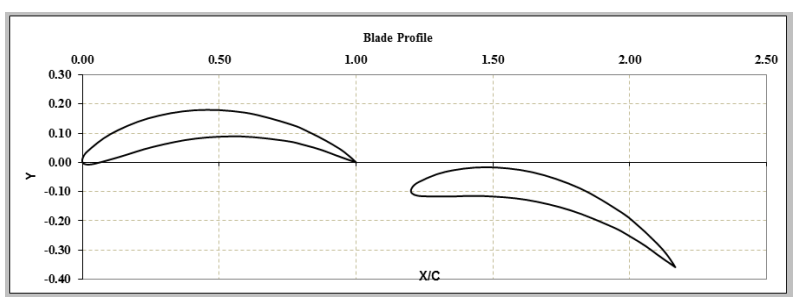

Figure 25a. Relative positions of tandem blades, $a=0.2, t=-0.1$, rear blade stagger angle $\lambda=15^{\circ}$.

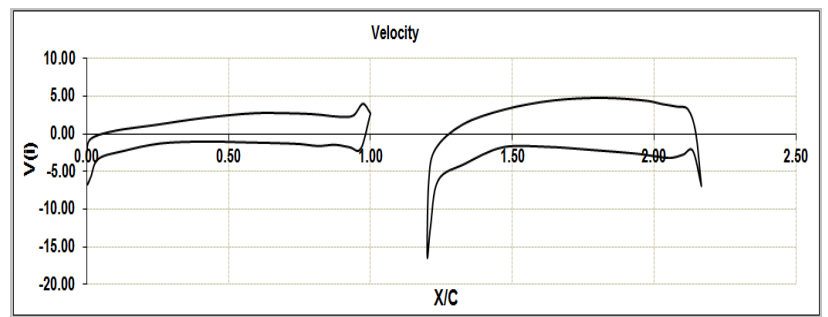

Figure 25b. Velocity distributions, $a=0.2, t=-0.1$, rear blade stagger angle $\lambda=15^{\circ}$.

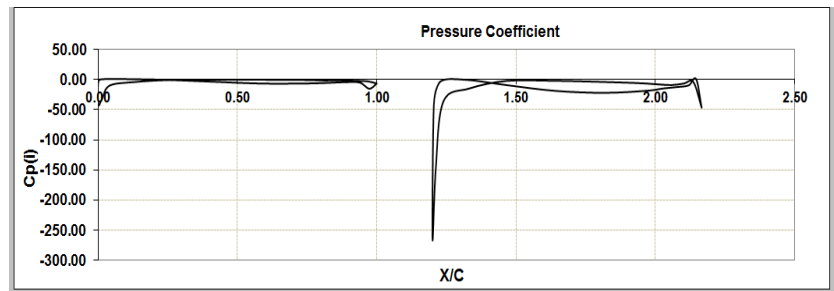

Figure 25c. Distributions of pressure coefficient, $a=0.2, t=-0.1$, rear blade stagger angle $\lambda=15^{\circ}$.

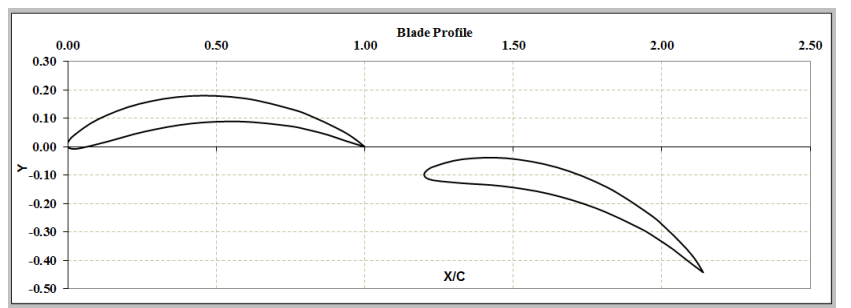

Figure 26a. Relative positions of tandem blades, $a=0.2, t=-0.1$, rear blade stagger angle $\lambda=20^{\circ}$. 


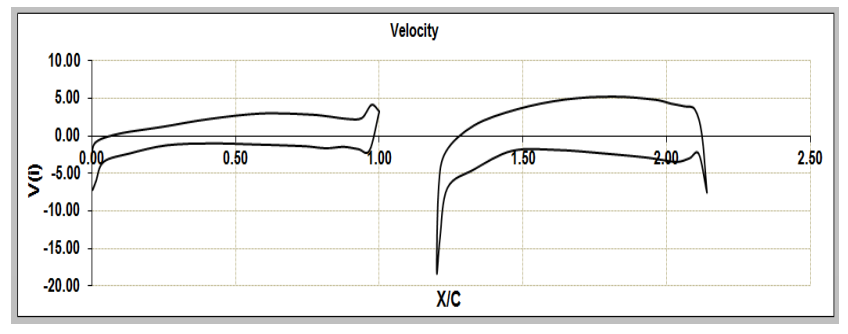

Figure 26b. Velocity distributions, $a=0.2, t=-0.1$, rear blade stagger angle $\lambda=20^{\circ}$.

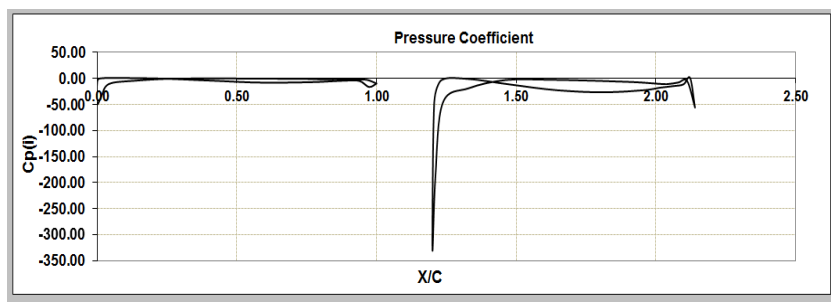

Figure 26c. Distributions of pressure coefficient, $a=0.2, t=-0.1$, rear blade stagger angle $\lambda=20^{\circ}$.

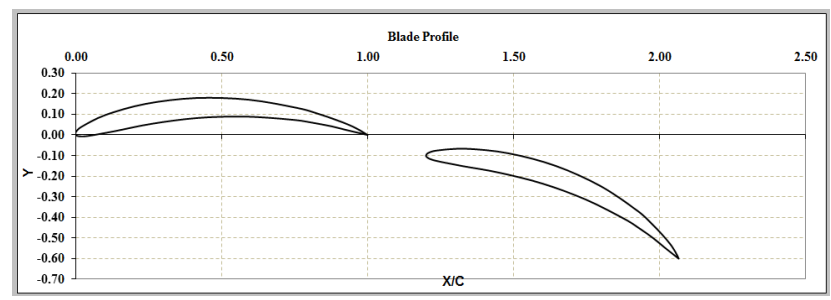

Figure 27a. Relative positions of tandem blades, $a=0.2, t=-0.1$, rear blade stagger angle $\lambda=30^{\circ}$.

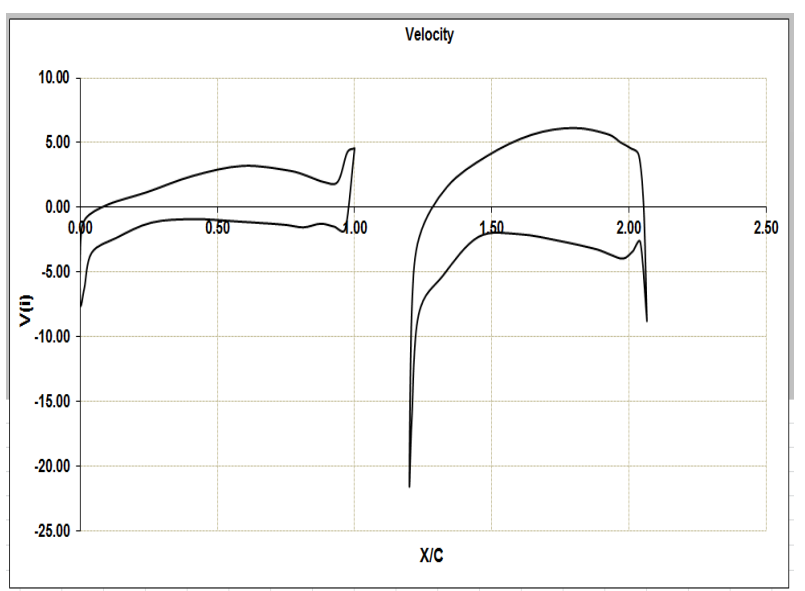

Figure 27b. Velocity distributions, $a=0.2, t=-0.1$, rear blade stagger angle $\lambda=30^{\circ}$.

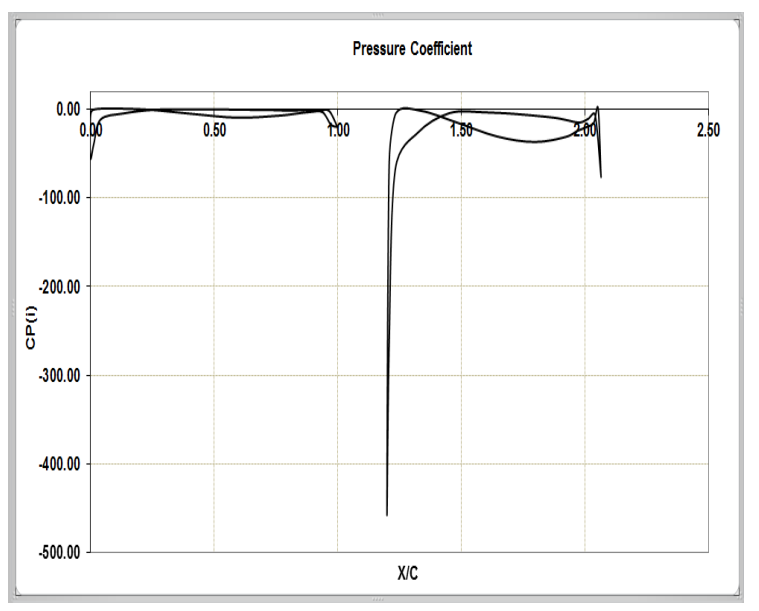

Figure 27c. Distributions of pressure coefficient, $a=0.2, t=-0.1$, rear blade stagger angle $\lambda=30^{\circ}$.

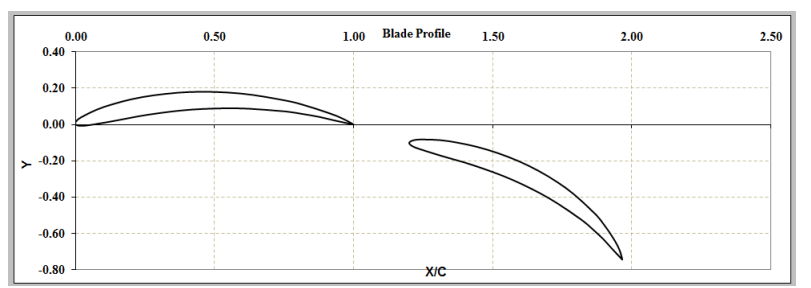

Figure 28a. Relative positions of tandem blades, $a=0.2, t=-0.1$, rear blade stagger angle $\lambda=40^{\circ}$.

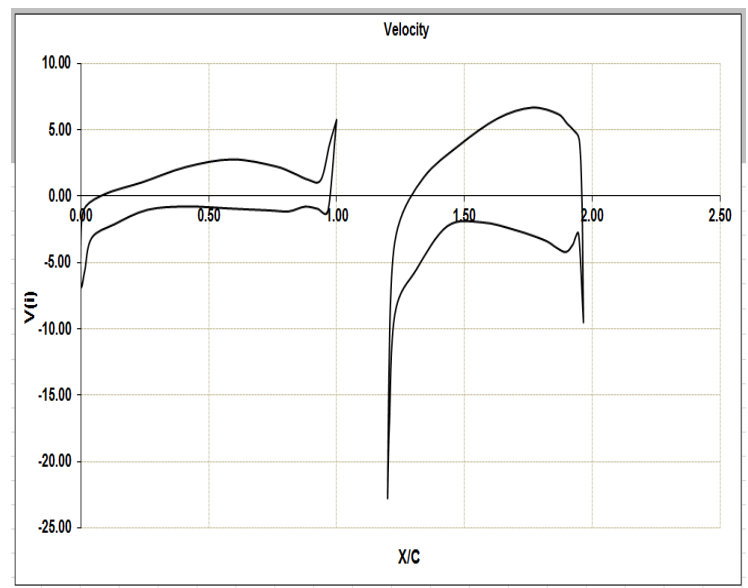

Figure 28b. Velocity distributions, $a=0.2, t=-0.1$, rear blade stagger angle $\lambda=40^{\circ}$. 


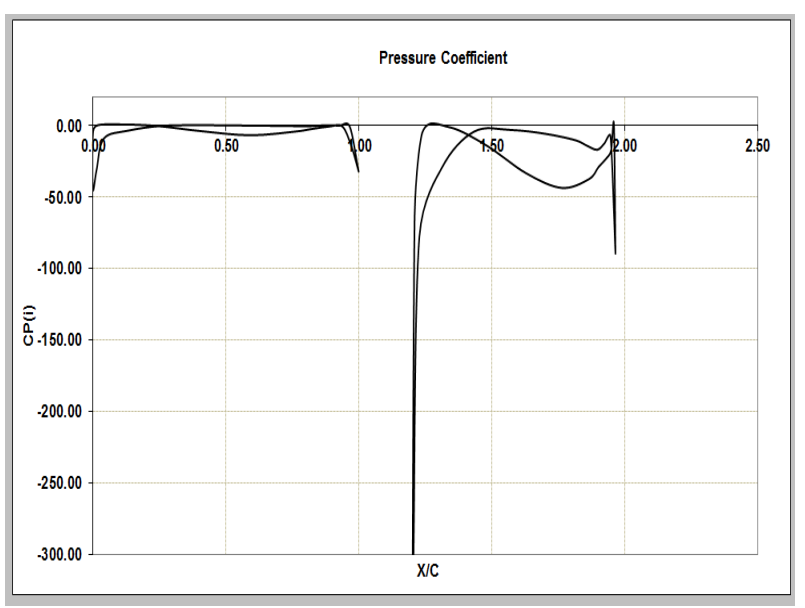

Figure 28c. Distributions of pressure coefficient, $a=0.2, t=-0.1$, rear blade stagger angle $\lambda=40^{\circ}$

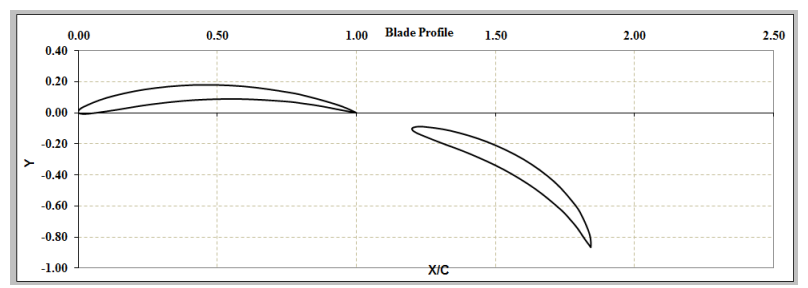

Figure 29a. Relative positions of tandem blades, $a=0.2, t=-0.1$, rear blade stagger angle $\lambda=50^{\circ}$.

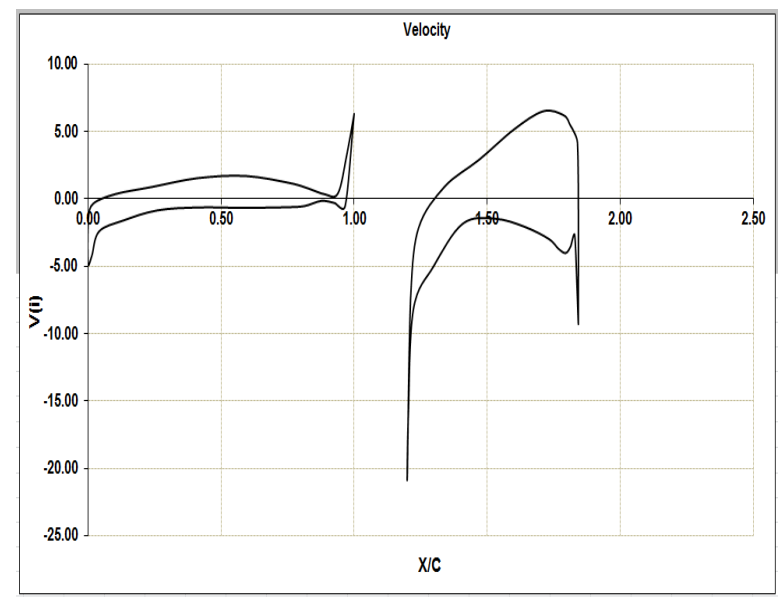

Figure 29b. Velocity distributions, $a=0.2, t=-0.1$, rear blade stagger angle $\lambda=50^{\circ}$.

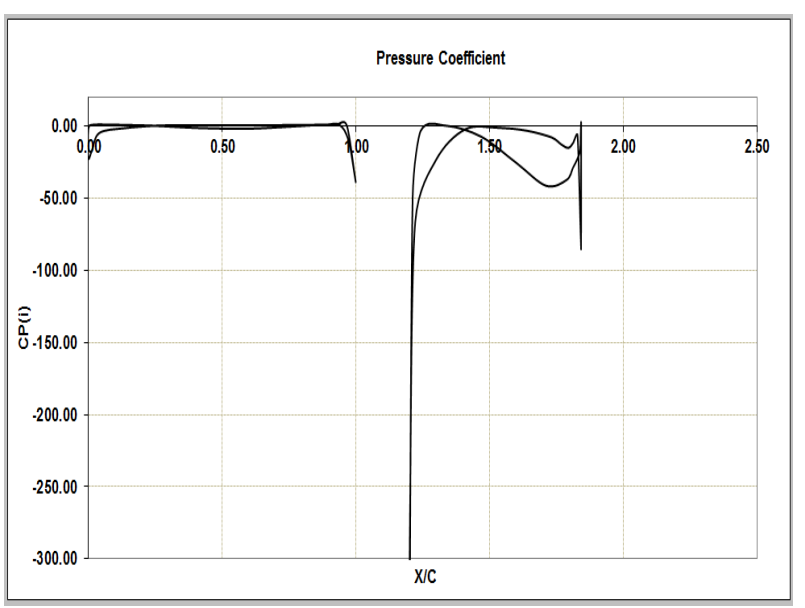

Figure 29c. Distributions of pressure coefficient, $a=0.2, t=-0.1$, rear blade stagger angle $\lambda=50^{\circ}$.

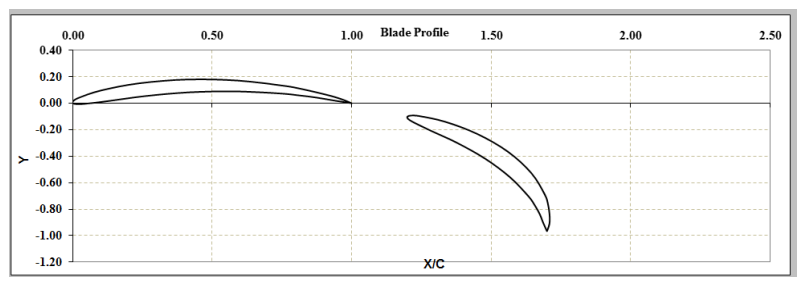

Figure 30a. Relative positions of tandem blades, $a=0.2, t=-0.1$, rear blade stagger angle $\lambda=60^{\circ}$.

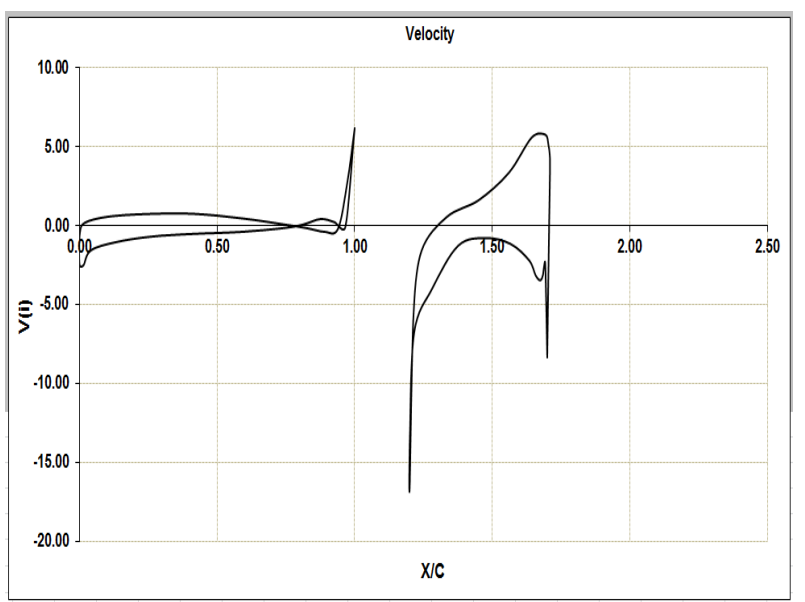

Figure 30b. Velocity distributions, $a=0.2, t=-0.1$, rear blade stagger angle $\lambda=60^{\circ}$. 


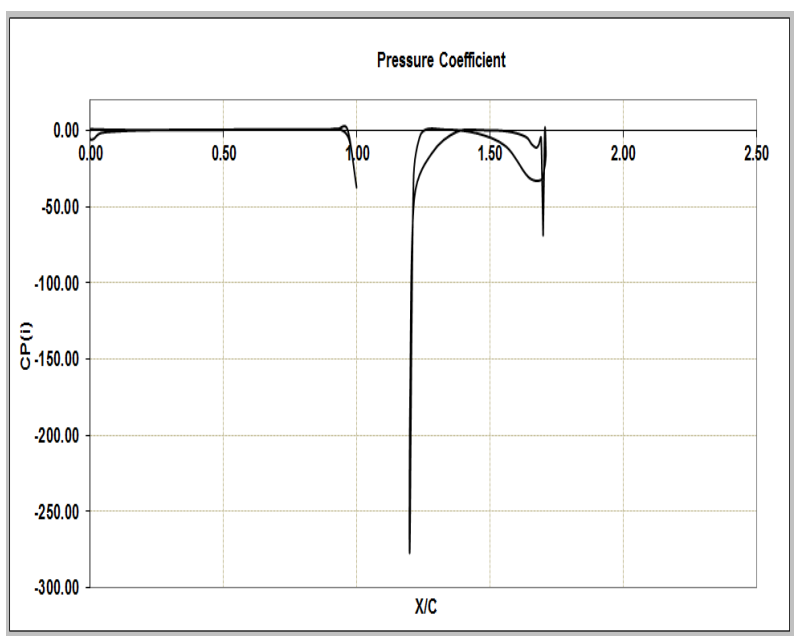

Figure 30c. Distributions of pressure coefficient, $a=0.2, t=-0.1$, rear blade stagger angle $\lambda=60^{\circ}$.

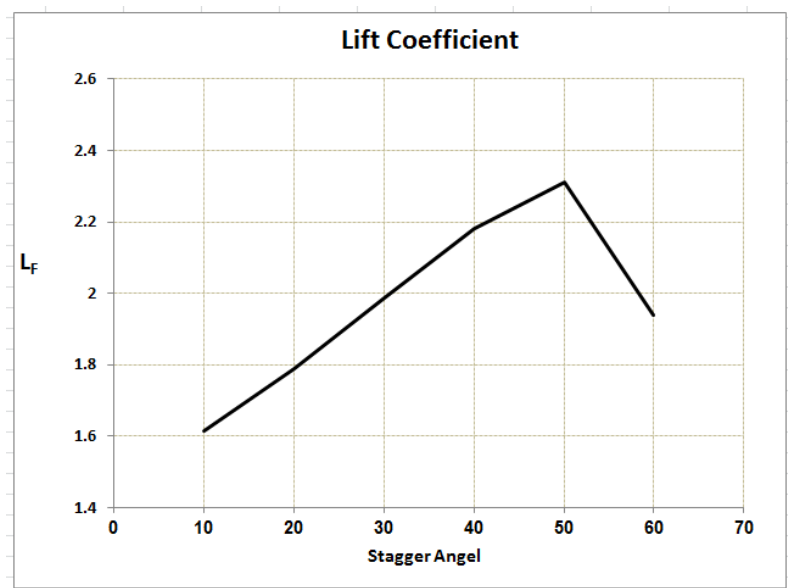

Figure 31. Lift coefficient variation of the rear Blade with its stagger angle.

Figure 32a illustrates velocity distribution on front blade for different staggered angles of the rear blade, "No gap nozzle effect". It is evident that the flow is highly influenced by the change in stagger angle of the rear blade. This influence is presented as a blockage effect that causes a lower flow velocity in the proximity of the trailing edge on the pressure surface with increasing the stagger angle. It is also shown that the lowest velocity on the pressure surface occurs when the rear blade is set at a stagger angle of $50^{\circ}$. This is due to the increase of the blockage effect. Generally, on the suction surface, the velocity increases with the increase in stagger angle of the rear blade.

Figure $32 \mathrm{~b}$ shows velocity distribution along the rear blade. On the pressure surface the velocity decreases with the increase in stagger angle of the rear blade. With the increase in the stagger angle, the inlet flow angle to the rear blade increases. On the suction surface, the contrary to the pressure surface occurs. The velocity increases with the increase of the stagger angle of the rear blade and reaches a maximum value at a stagger angle of $50^{\circ}$.

Figure 33a shows the pressure distribution on front blade for different stagger angles of the rear blade, "No gap nozzle effect". On the pressure surface, it is shown that pressure increases with increasing the stagger angle of the rear blade due to the decrease in velocity on that surface as explained previously in Figure 32a. While on the suction surface, the pressure increases by small amounts with increasing in stagger angle.

Figure $33 \mathrm{~b}$ demonstrates the pressure distribution on rear blade for different stagger angles of the rear blade, "No gap nozzle effect". The pressure distributions on both the pressure and suction surfaces have the same pattern of the front blade except for the increase in pressure limits of the rear blade as compared to the front blade.

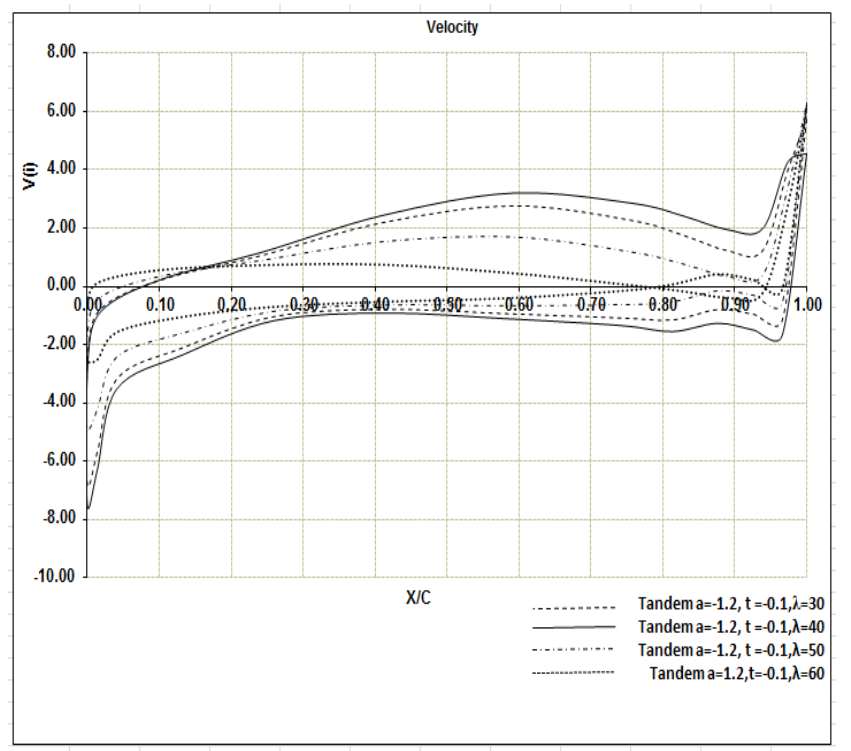

Figure 32a. Velocity distribution on front blade for different stagger angles of the rear blade, "No gap nozzle effect".

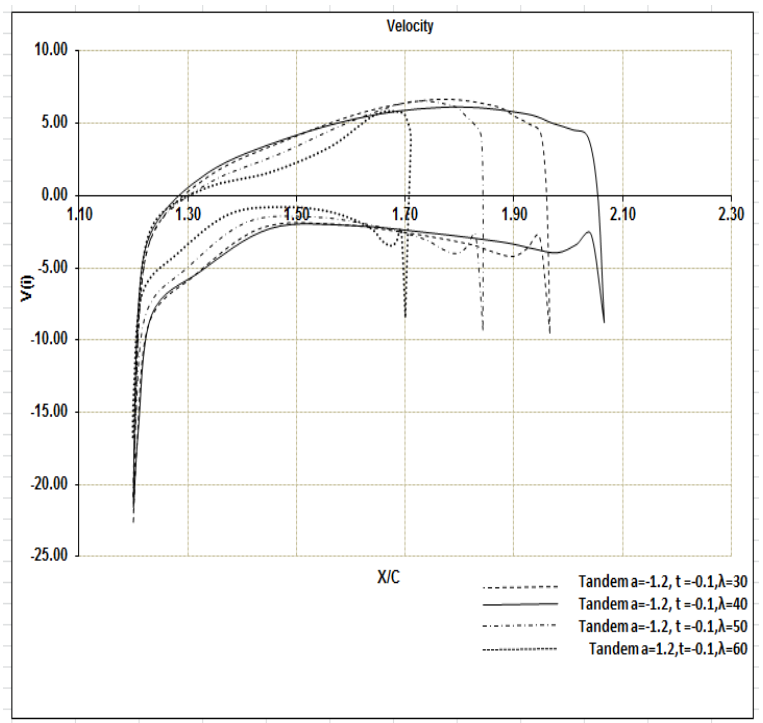

Figure 32b. Velocity distribution on rear blade for different stagger angles of the rear blade, "No gap nozzle effect". 


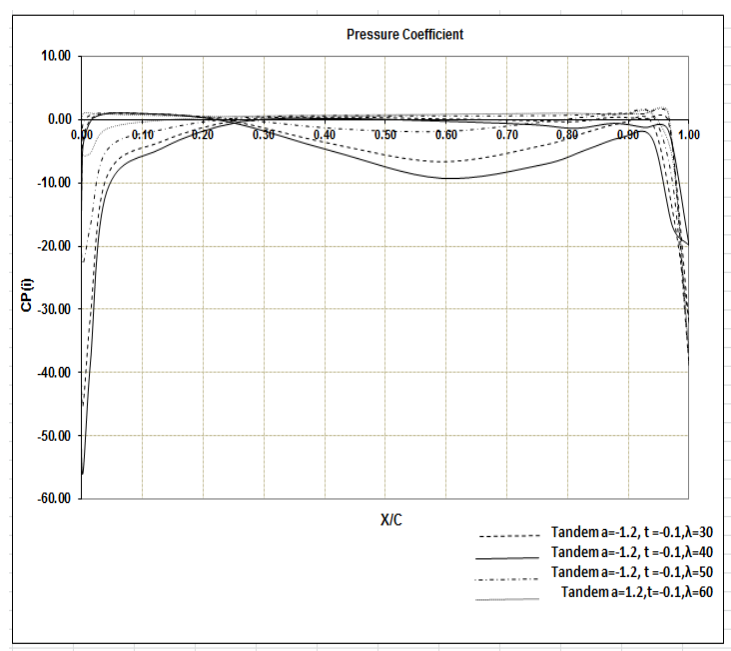

Figure 33a. Pressure distribution on front blade for different stagger angles of the rear blade, "No gap nozzle effect".

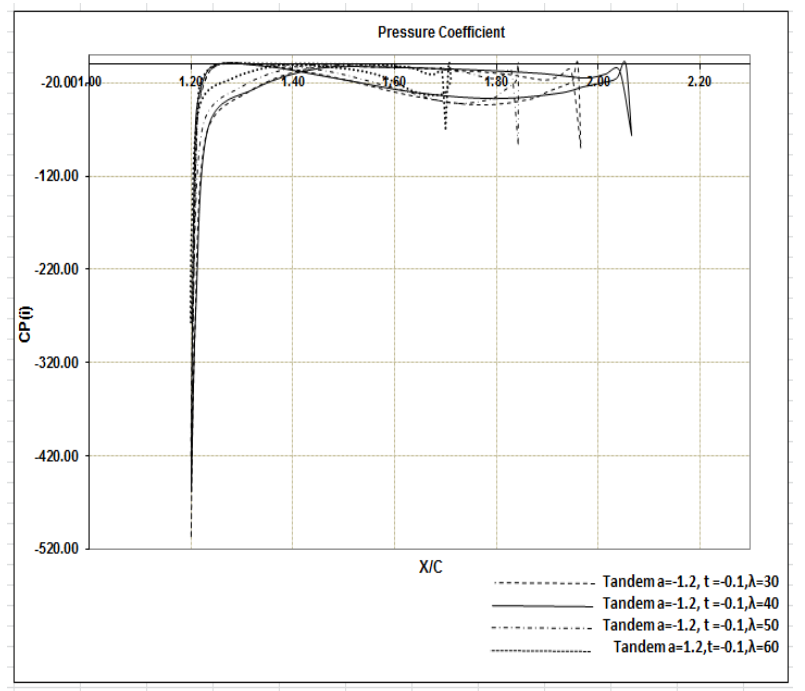

Figure 33b. Pressure distribution on rear blade for different stagger angles of the rear blade, "No gap nozzle effect".

\subsection{Gap Nozzle Effect}

\subsubsection{Geometry Configuration}

The gap nozzle effects appear in the tandem blades when the rear blade is positioned in the vicinity of the trailing edge of the front blade. The zone between the blades can be represented as a convergent gap with an inlet and outlet area characterized by the distances $F 1$ and $F 2$, respectively, Figure 34.

Therefore, the relation $F 1 / F 2$ gives a measure of the flow acceleration by the presence of the nozzle configuration. In this section the ratio $F 1 / F 2$ is used as a characteristic parameter for each test case of tandem blades.

The tangential displacements that satisfy the condition fixed by the gap nozzle inlet and outlet ratio were calculated graphically using Excel. An example of the procedure is depicted in Figure 35 for tandem blades with $F 1 / F 2$ equal to 2.2 and axial distance of -0.87 . In the Figure 35, the value of $F 1=0.11$ and $F 2=0.05$, thus the ratio is $F 1 / F 2=2.2$.

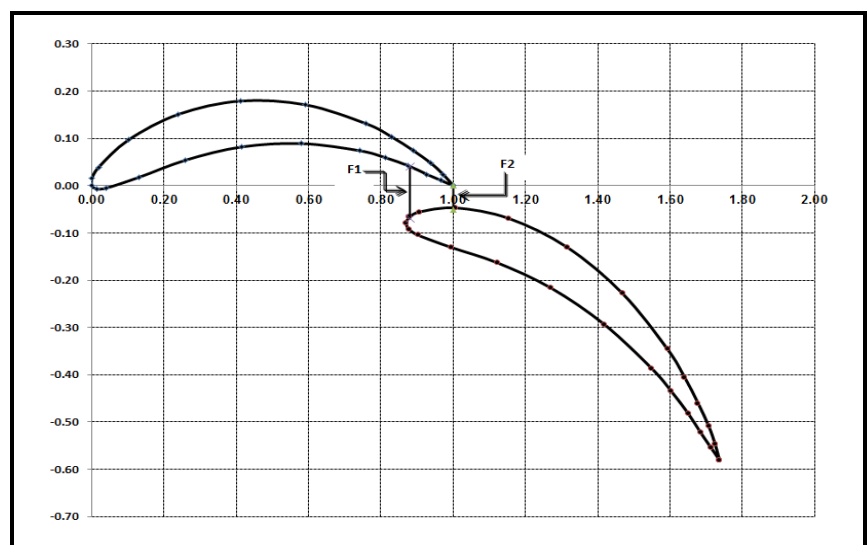

Figure 34. Geometry configuration, "Gap nozzle effects".

This part of the investigation was conducted to assess the advantage of a tandem cascade with the presence of gap nozzle geometry between the blades as opposed to the two single blades acting independently. The study is made on the bases of the static pressure distribution along the blades and the lift coefficient. The final gap-nozzle parameters that describe the area between the profiles are summarized in Table 4 for the various tested cases.

Table 4 Gap nozzle effect, tandem blades

\begin{tabular}{lll}
\hline Axial displacement $[\boldsymbol{a}]$ & Tangential displacement $[\boldsymbol{t}]$ & $\boldsymbol{F 1 / F 2}$ \\
\hline \multirow{3}{*}{0.87} & -0.08 & 2.2 \\
& -0.1 & 1.86 \\
& -0.06 & 3.33 \\
\hline
\end{tabular}

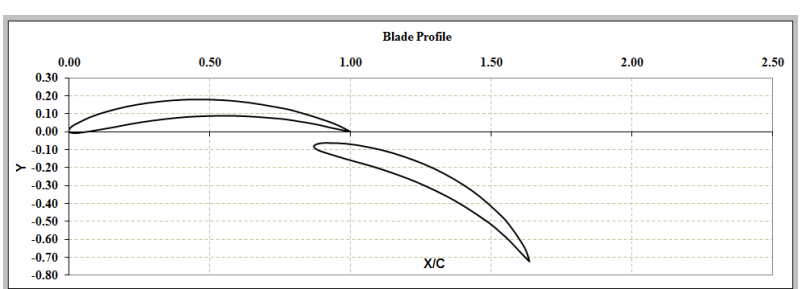

Figure 35a. Relative positions of tandem blades, $a=-0.87, t=-0.08$, rear blade angle relative to front blade $=40, F 1 / F 2=2.2$.

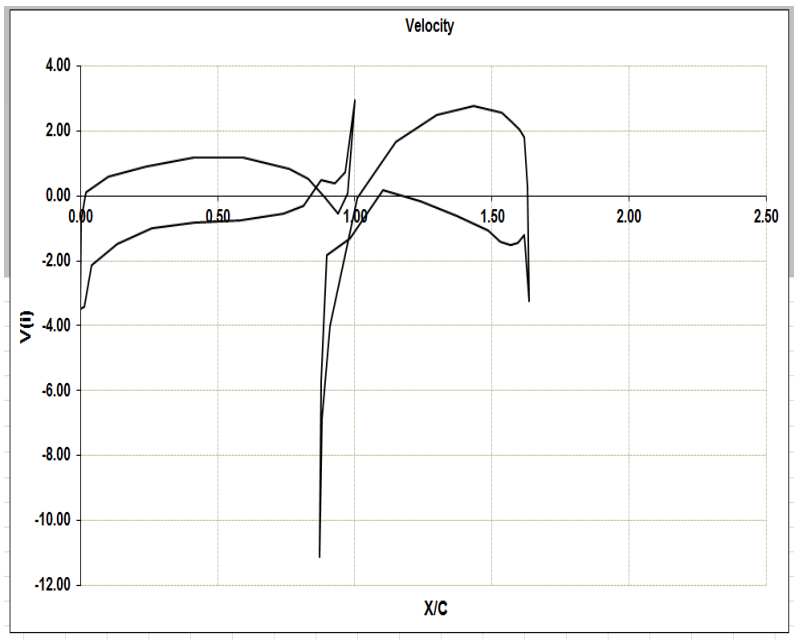

Figure 35b. Velocity distributions of tandem blades, $a=-0.87, t=-0.08$, rear blade stagger angle $=40, F 1 / F 2=2.2$. 


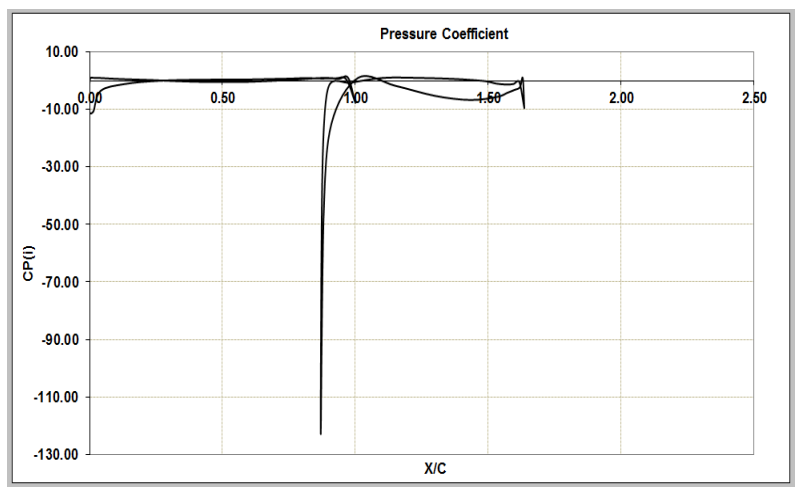

Figure 35c. Pressure distributions of tandem blades, $a=-0.87, t=-0.08$, rear blade stagger angle $=40, F 1 / F 2=2.2$.

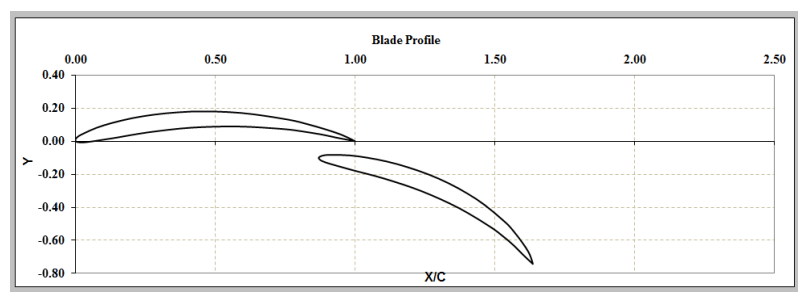

Figure 36a. Relative positions of tandem blades, $a=-0.87, t=-0.1$, rear blade angle relative to front blade $=40, F 1 / F 2=1.86$.

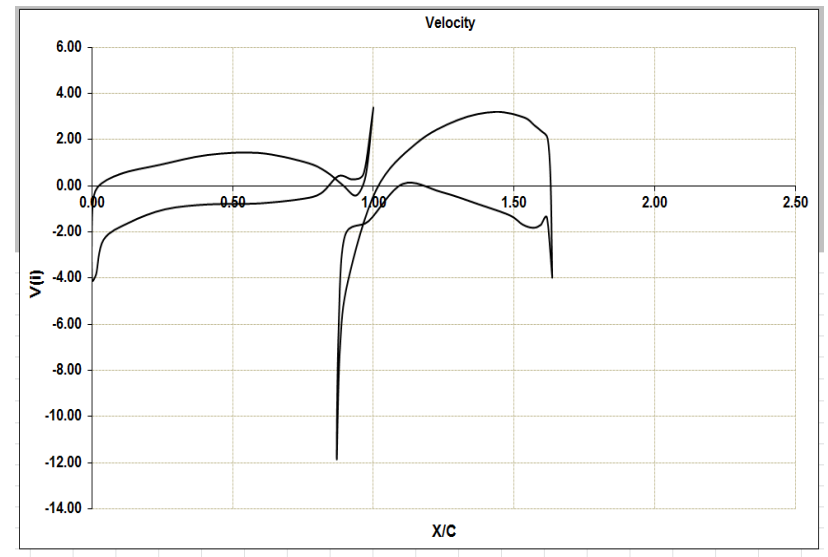

Figure 36b. Velocity distributions of tandem blades, $a=-0.87, t=-0.1$, rear blade stagger angle $=40, F 1 / F 2=1.86$.

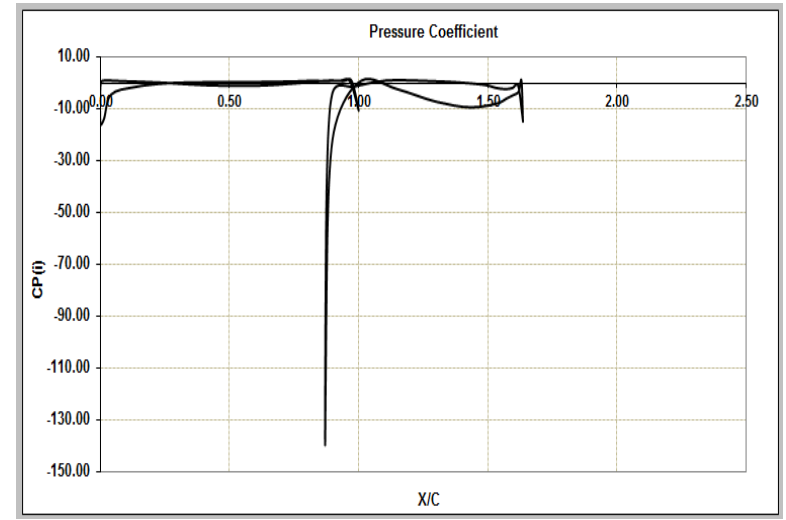

Figure 36c. Pressure distributions of tandem blades, $a=-0.87, t=-0.1$, rear blade stagger angle $=40, F 1 / F 2=1.86$.

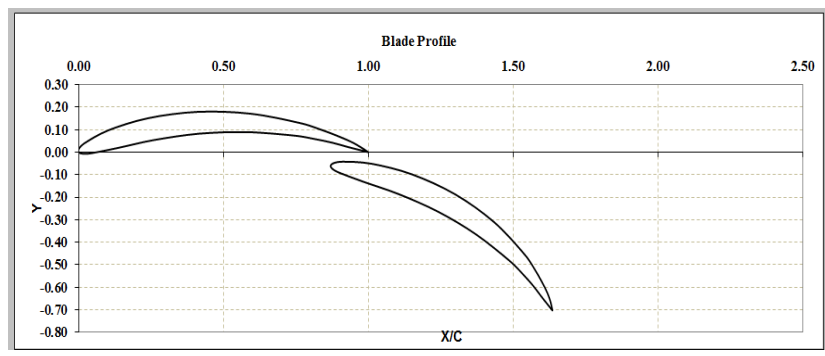

Figure 37a. Relative positions of tandem blades, $a=-0.87, t=-0.06$, rear blade angle relative to front blade $=40, F 1 / F 2=3.33$.

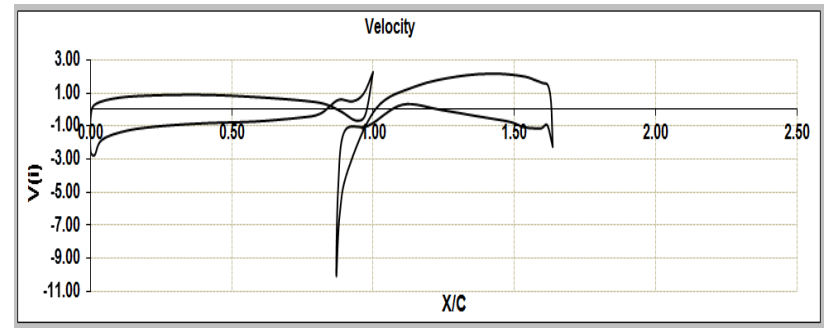

Figure 37b. Velocity distributions of tandem blades, $a=-0.87, t=-0.06$, rear blade stagger angle $=40, F 1 / F 2=3.33$.

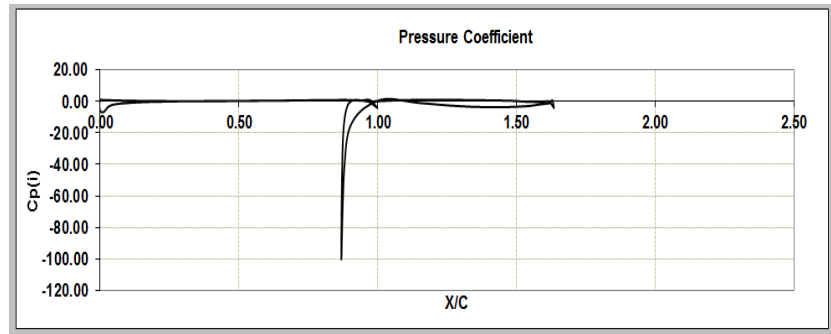

Figure 37c. Pressure distributions of tandem blades, $a=-0.87, t=-0.06$, rear blade stagger angle $=40, F 1 / F 2=3.33$.

\subsubsection{Test Cases}

From the results of Figures 35-37, it is noticed that the relative position of the rear blade does not disturb the flow along the suction surface of the front blade. This behavior is expected because the interaction between the two blades is inexistent in this surface. Thus, the flow behavior is quite similar to the flow along the blade acting alone.

So, it is noticed that there is an initial acceleration up to $60 \%$ of the chord from the leading edge; then, the flow starts decelerating in the range from $0.6<\mathrm{x} / \mathrm{C}<0.92$, followed by steep diffusion towards the trailing edge.

Nevertheless, it is appreciable that if the tangential displacement of the rear blade is quite large, which means low values of $F 1 / F 2$ ratio, the flow is slightly more decelerated than the corresponding flow on the suction surface of a rear blade with no gap nozzle. This fact is attributed to a mass flow balance between the gap nozzle area, where the flow has low momentum, and the flow channel between the two consecutive tandem blades.

On the pressure surface of the front blade, it is evident that the flow is highly influenced by the gap nozzle area. This influence is presented as a blockage effect that causes an increase in flow velocity in the proximity of the front blade trailing edge. 
Figure 38a illustrates a comparison between the various values of $F 1 / F 2$ for the front blade with a $=-0.2$. It is clear that the lowest velocity on the pressure surface occurs when the ratio $F 1 / F 2$ is the highest. It is also appreciable that when the ratio $F 1 / F 2$ increases, there is an increment in the blockage effect. The flow behavior, on this surface, shows a constant deceleration up to the gap channel limit. Thereafter, the flow enters into the gap nozzle area and the velocity increases towards the trailing edge of the blade. This behavior is due to the nozzle effect in the gap zone.

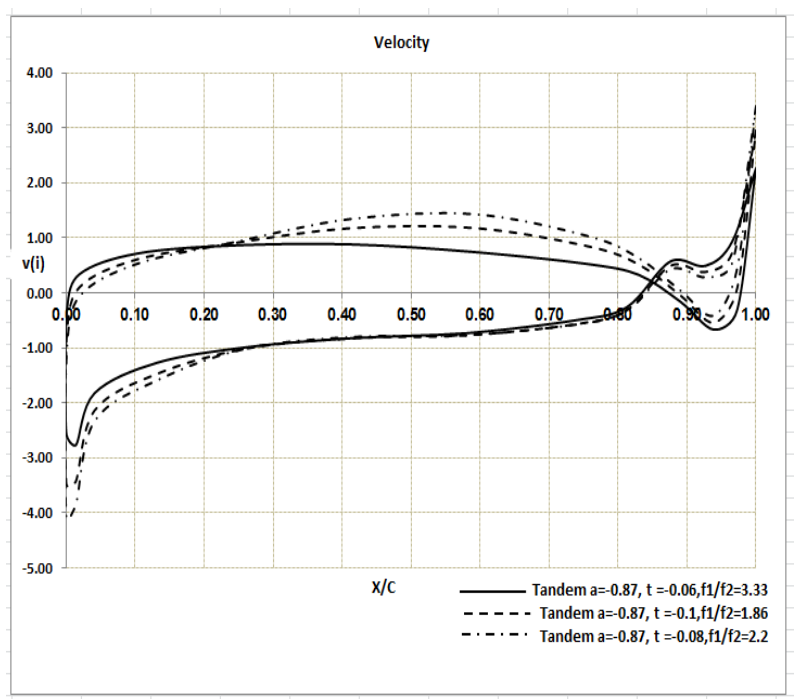

Figure 38a. Velocity distribution on front blade for different arrangements, "Gap nozzle effect".

Figure $38 \mathrm{~b}$ shows that, on the pressure surface of the rear blade and for high values of $F 1 / F 2$, the flow is forced to go along the pressure surface of the rear blade due to the blockage phenomenon in the gap nozzle area, which is presented as a decrement of the flow peak velocity.

Thus, the momentum transfer on the suction surface of the rear blade is not sufficient to ensure the no presence of flow disturbances. The low-momentum flow at the inlet of the gap zone is responsible for the flow separation at the rear part of the rear blade. Thus, an increase of the total losses is expected because the blade operates in stall conditions.

Concerning the pressure distribution on the front blade, Figure $39 \mathrm{a}$, it is demonstrated that, as seen before in the velocity distribution, the relative position of the rear blade does not disturb the flow along the suction surface of the front blade. Thus, the flow behavior is quite similar to the flow along the blade acting alone. On the suction surface, the flow is slightly more accelerated with the larger values of the tangential displacement of the rear blade, i.e., lower values of $F 1 / F 2$. Also, the flow is more accelerated for all cases with "gap nozzle effect" in comparison to the corresponding flow on the suction surface of tandem blades with "no gap nozzle effect". This is due to the blockage effect; i.e., as the ratio $F 1 / F 2$ increases the blockage effect increases.

Figure $39 \mathrm{~b}$ shows the pressure distribution on the rear blade. On the pressure surface, for high values of $F 1 / F 2$, the flow is forced to go along the pressure surface due to the blockage phenomenon, which is presented as a decrement of the flow peak velocity in the gap nozzle area.

For all cases, the gap-nozzle geometry prevents high flow acceleration at the suction surface of the rear profile. Therefore, it is said that the peak velocity of the rear blade is influenced by the relative position of the blades in a tandem cascade. In all cases, the flow is characterized by an initial acceleration, which is limited by the nozzle geometry, up to the gap-channel influence limit. Thereafter, a constant deceleration towards the trailing edge is evident.

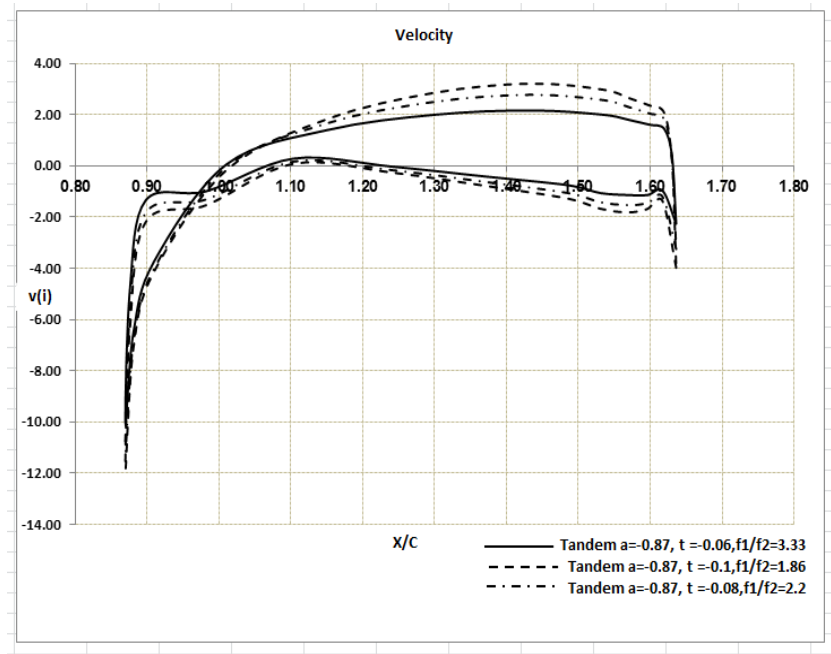

Figure 38b. Velocity distribution on rear blade for different arrangements, "Gap nozzle effect".

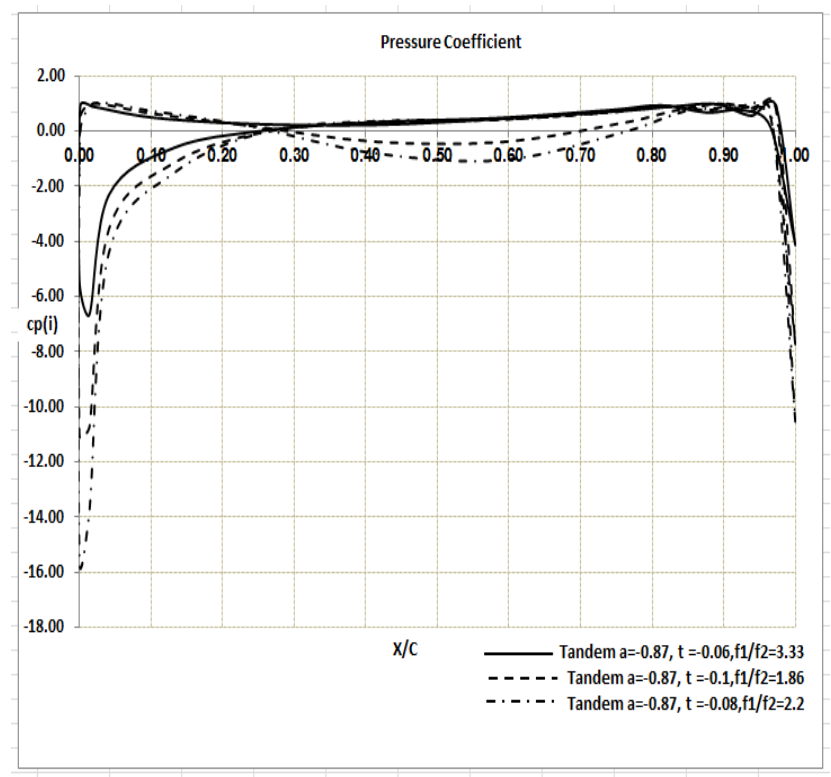

Figure 39a. Pressure distribution on front blade for different arrangements, "Gap nozzle effect". 


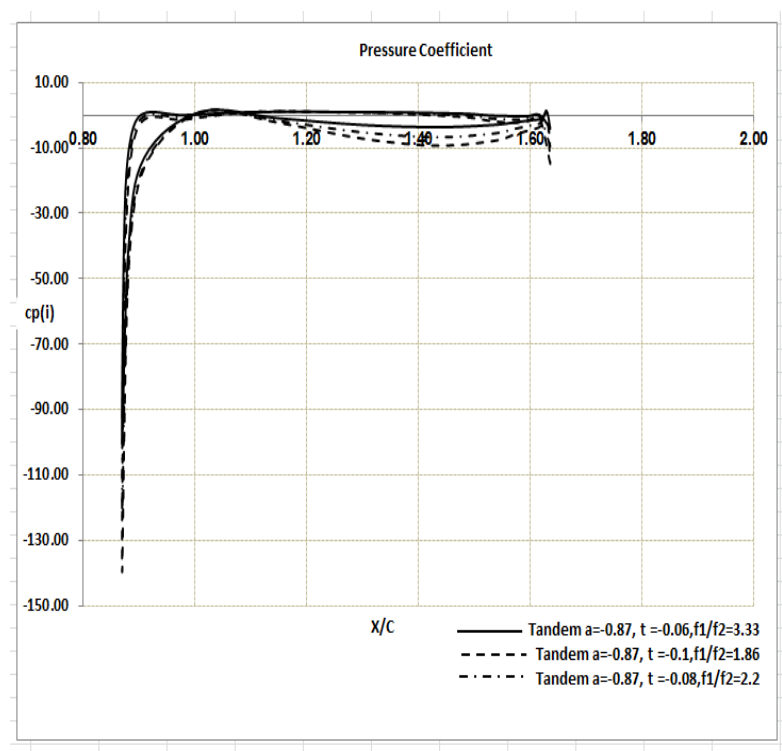

Figure 39b. Pressure distribution on rear blade for different arrangements, "Gap nozzle effect".

Figure 40 illustrates the lift coefficient of rear blade at different gap nozzles between the front and rear blade. In all cases, it is noticed that the gap-nozzle geometry highly influences the peak velocity on the suction surface of the rear blade. It is also concluded that if the rear blade is located in such a way that the gap-nozzle geometry promotes sufficient flow guidance for efficient momentum transfer on the rear blade suction surface, the rear blade shows higher lift.

This is very clear for the cases where the gap-nozzle area is 1.86 and 2.22. In these two cases, the gap-nozzle area promotes sufficient guidance on the rear blade that results in a higher pressure difference across the blade, Figure 39. However, the opposite occurs for gap-nozzle area of 3.33 , which does not give enough flow guidance on the rear blade. Thus, lower pressure difference distribution is found and consequently, lower lift is obtained in comparison to the two cases that were mentioned previously.

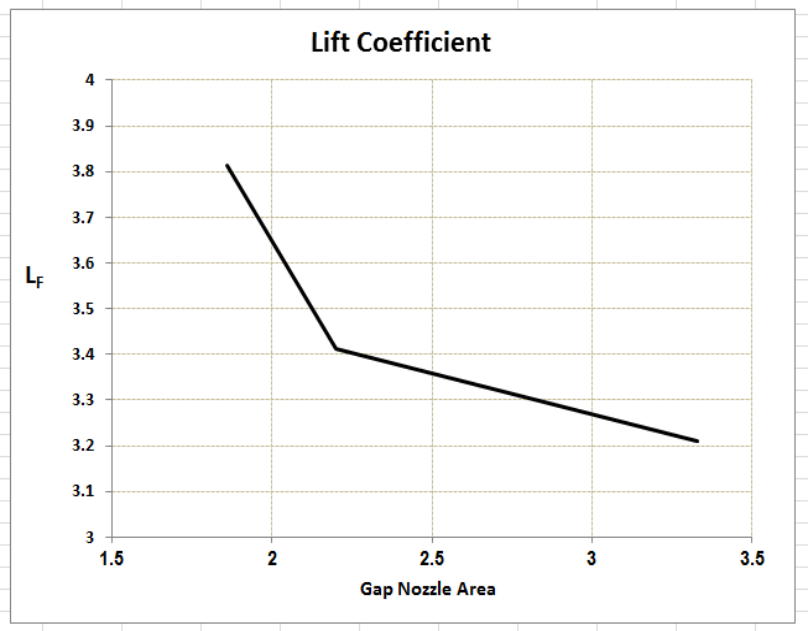

Figure 40. Lift coefficient of rear blade at different gap nozzles between the front and rear blade.

\section{Conclusions}

In this study, a numerical investigation of the steady two-dimensional flow for compressor tandem blades was carried out. The interaction mechanism between the two blades was inspected by varying the relative position of the two blades. Based on the above results and discussions, the following conclusions are obtained:

1. Although, it is not shown in the paper, validation of the present model and methodology was carried out with the study of [14]. Based on comparisons between the present results and those of [14], it was clear that the present model and methodology are suitable for the present study.

2. The deflection capability of tandem blades is higher than that of a single blade. This increase of deflection angle is attributed to the no-presence of flow separation with the increase of loading.

3. If the rear blade is positioned in the wake of the front blade (no gap nozzle effect), there is a decrease in lift coefficient as a consequence of the velocity deficit that causes flow disturbances on the suction surface of the rear blade.

4. When the rear blade is located in the viscous free region and there is "no gap nozzle effect", the tandem blades show an increase in the lift coefficient. This is a consequence of the interaction between the wake of the first blade and the velocity field in the vicinity of the suction surface of the rear blade.

5. In all cases, it was noticed that the gap nozzle geometry highly influences the peak velocity on the suction surface of the rear blade due to the accelerated flow in the gap nozzle channel.

6. If the second blade is located in such a way that the gap nozzle geometry promotes sufficient flow guidance for efficient momentum transfer on the suction surface of the rear blade, the rear blade shows higher lift than the case of two blades with "no gap nozzle effect".

7. The presence of the rear blade in the proximity of the pressure surface of the front blade causes a decrement in the flow velocity. This decrement is characterized as a blockage effect that increases with the increase of the ratio $F 1 / F 2$ and vice versa.

8 . For higher values of $F 1 / F 2$, the gap nozzle geometry does not promote sufficient flow guidance for efficient momentum transfer on the suction surface of the rear blade. Therefore, the lift decreases for higher values of $F 1 / F 2$. On the other hand, when the rear blade is positioned in such a way that the gap nozzle energizes the wake of the front profile and promotes sustained flow attachments on the suction surface of the rear blade (the wake having low momentum is filled up by the high momentum flow near the suction surface of the rear blade), the lift increases. 


\section{Nomenclature}

$\begin{array}{ll}a & \text { Axial distance } \\ C & \text { Chord } \\ C_{l} & \text { Chord length of front blade } \\ C_{2} & \text { Chord length of rear blade } \\ C_{l} & \text { Lift coefficient } \\ C_{p} & \text { Pressure coefficient } \\ C_{t} & \text { Overall chord length } \\ F_{1} & \text { Inlet gap distance } \\ F_{2} & \text { Outlet gap distance } \\ L & \text { Lift } \\ \dot{m} & \text { Mass flow rate } \\ P_{s h a f t} & \text { Shaft power } \\ S & \text { Blade spacing } \\ T & \text { Tangential displacement } \\ U & \text { Velocity in } x \text {-direction } \\ U_{l} & \text { Blade speed at radius } r_{l} \\ U_{2} & \text { Blade speed at radius } r_{2} \\ V & \text { Velocity in } y \text {-direction } \\ V_{\theta 1} & \text { tangential velocity at radius } r_{l} \\ V_{\theta 2} & \text { tangential velocity at radius } r_{2} \\ W_{l} & \text { Inlet velocity } \\ W_{l 2} & \text { Inlet velocity at rear blade } \\ W_{2} & \text { Outlet velocity }\end{array}$

\section{Greek}

$\begin{array}{ll}\alpha & \text { Attack angle } \\ \beta & \text { Rear blade relative angle to front blade } \\ \beta_{1} & \text { Relative inflow angle } \\ \beta_{2} & \text { Relative outflow angle } \\ \delta & \text { Deviation angle } \\ \gamma & \text { Vortex strength } \\ \Gamma & \text { Circulation } \\ \lambda & \text { Stagger angle } \\ \rho & \text { Density } \\ \theta & \text { Camber angle }\end{array}$

\section{Abbreviations}

FB

Front blade

$\mathrm{RB}$

\section{References}

[1] A. J. Wennerstrom, "Low aspect ratio axial flow compressors: why and what it means," ASME Journal of Turbomachinery, Vol. 111, pp. 357-365, 1989.

[2] A. K. Saha, and B. Roy, "Experimental analysis of controlled diffusion compressor cascades with single and tandem airfoils," ASME paper number 95-CTP-41, 1995.

[3] A. K. Saha, and B. Roy, "Experimental investigations on tandem compressor cascade performance at low speeds," Experimental Thermal and Fluid Science, Vol. 14, pp. 263-276, 1997.

[4] E. Sheets, "Multiple row blades for blowers," ASME paper number 88-GT-124, 1988.

[5] A. Weber, and W. Steinert, "Design, Optimization, and Analysis of a High-Turning Transonic Tandem Compressor Cascade," ASME paper number 97-GT-412, 1997.

[6] General Electric Co. Manuals, Al-shabab Power Plant (1000 MW-simple gas turbine plant), Ismailia, Egypt.

[7] K. Bammert, and H. Beelte, "Investigations of an axial flow compressor with tandem cascades," ASME Journal of Engineering Power, pp. 971-977, October 1980.

[8] K. Bammert, and R. Staude, "Optimization for rotor blades of tandem design for axial flow compressors," ASME Journal of Engineering for Power, pp. 369-375, April 1980.

[9] J. McGlumphy, 2-D computational studies of subsonic axial rotors incorporating dual airfoils, UTSR Project Report for summer 2005.

[10] J. McGlumphy, W.-F. Ng, S. R. Wellborn, and S. Kempf, "Numerical investigation of tandem airfoils for subsonic axialflow compressor blades," Journal of Turbomachinery, Vol. 131, pp. 1-8, April 2009.

[11] J. McGlumphy, W.-F. Ng, S. R. Wellborn, and S. Kempf, "3D numerical investigation of tandem airfoils for a core compressor rotor," Journal of Turbomachinery, Vol. 132, pp. $1-9$, July 2010.

[12] G. A. Canon, Numerical investigation of the flow in tandem compressor cascade, Diploma Thesis, Vienna University of Technology, Austria, 2004.

[13] J. Katz, and A. Plotkin, Low-Speed Aerodynamics, 2nd ed., Cambridge University Press, 2001.

[14] C. A. Cox, Two Element Linear Strength Vortex Panel Method, Senior Project, Faculty of the Aerospace Engineering, California Polytechnic State University, San Luis, USA, March 2011. 